ESAIM: PROCEEDINGS, December 2012, Vol. 38, p. 409-428

F. Coquel, M. Gutnic, P. Helluy, F. Lagoutière, C. Rohde, N. Seguin, Editors

\title{
CONSERVATION LAWS WITH A NON-LOCAL FLOW APPLICATION TO PEDESTRIAN TRAFFIC.
}

\author{
Magali LÉcureuX-Mercier ${ }^{1}$
}

\begin{abstract}
In this note, we introduce some models of pedestrian traffic and prove existence and uniqueness of solutions for these models.

Résumé. On présente ici différents modèles de trafic piéton et on prouve l'existence et l'unicité des solutions pour ces modèles.
\end{abstract}

\section{INTRODUCTION}

In the last decades, the crowds' dynamics has attracted a lot of scientific interest. A first reason of this interest is to understand how crowd disasters happened in some panic events, for example at the end of football play, during concerts, in the case of fire, or in place of pilgrimage (e.g. on Jamarat Bridge in Saudi Arabia, see [13]). Another reason lies in architecture of buildings such as subway stations or stadiums, where a lot of pedestrians are crossing. The goals here are consequently twofold: in one hand we want to understand the behavior of pedestrians in panic and adapt the regulation of traffic in order to avoid deaths; in the other hand, we want to model the interaction of several kinds of pedestrians with different objectives and in particular study how the geometry influences the general pattern.

In a macroscopic setting, a population is described by its density $\rho$ which satisfies the conservation law

$$
\partial_{t} \rho+\operatorname{Div}(\rho V(t, x, \rho))=0, \quad \rho(0, \cdot)=\rho_{0},
$$

where $V(t, x, \rho)$ is a vector field describing the velocity of the pedestrians depending on the time $t \geq 0$, the space $x \in \mathbb{R}^{N}$ and the density $\rho$. According to the choice of $V$, various behaviors can be observed. Several authors already studied pedestrian traffic in two dimensions space $(N=2)$. Some of these models are local in $\rho$, that is to say $V$ depends on the local density $\rho(t, x)[2,8,15,16,20,21]$; other models use not only the local density $\rho(t, x)$ but the entire distribution of $\rho$, for example they depend on the convolution product $\rho(t) * \eta[11,22]$. Here, in the line of preceding papers [4-7], we present nonlocal macroscopic models for pedestrian traffic, we study these models and compare their properties.

Our first aim is to model the behavior of pedestrians in different situations: crowd behaves indeed differently in panic or in a normal situation where courtesy rules do apply. We also introduce models in the case of a population interacting with an individual, and in the case of several populations with different objectives. For instance, we want to include in our study the case of two populations crossing in a corridor.

Second, we want to study the introduced models and prove existence and uniqueness of solutions under various sets of hypotheses. We will use two kinds of arguments: the first one comes from Kružkov theory [17, 18], the

1 Technion - Israel Institute of Technology, Amado Building, Haifa, 32000 Israel, mercier@tx.technion.ac.il.

(C) EDP Sciences, SMAI 2012 
second one from the optimal transport theory. We want to prove existence and uniqueness of solutions for the various models presented below. Let us concentrate on the case of pedestrians in panic:

$$
\partial_{t} \rho+\operatorname{Div}(\rho v(\eta * \rho) \vec{\nu}(x))=0 ; \quad \rho(0, \cdot)=\rho_{0} .
$$

All the proofs of existence and uniqueness in this note are based on the following idea: let us fix the nonlocal term and, instead of (1), we study the Cauchy problem

$$
\partial_{t} \rho+\operatorname{Div}(\rho v(r * \eta) \vec{\nu}(x))=0, \quad \rho(0)=\rho_{0},
$$

where $r$ is a given function. Then, we introduce the application

$$
\mathscr{Q}:\left\{\begin{array}{ccc}
r & \mapsto & \rho \\
X & \rightarrow & X
\end{array}\right\},
$$

where the space $X$ has to be chosen so that

(a): $X$ is equipped with a distance $d$ that makes $X$ complete;

(b): the application $\mathscr{Q}$ is well-defined: the solution $\rho \in X$ exists and is unique (for a fixed $r$ );

(c): the application $\mathscr{Q}$ is a contraction.

Once we have fulfilled these conditions, we can prove existence and uniqueness of a solution using a fixed point argument.

Note that, in the modelling of pedestrian traffic, the space dimension $N$ has to be equal to two, but our results are in fact true for all $N \in \mathbb{N}$. For instance, they can be adapted in dimension $N=3$ to model the behavior of fishes or birds. Hence, we keep here a general $N$, even if we essentially think to the case $N=2$.

This note is organized as follows: in Section 2, we describe some nonlocal models and their properties. In Section 3 we study one of these models through Kružkov theory and in Section 4 we study the same model through optimal transport theory.

\section{Pedestrian Traffic Modelling}

\subsection{One-Population model}

\subsubsection{Pedestrian in panic}

The first model we present corresponds to pedestrians in panic and was studied in $[6,7]$, in collaboration with R. M. Colombo and M. Herty. A panic phenomenon appears under special circumstances in crowded events. In these cases, the people are no longer rational and try, no matter how, to reach their target. Let us denote $\rho(t, x)$ the density of pedestrians at time $t$, and position $x \in \mathbb{R}^{N}$, and consider the Cauchy problem (1). Here, $v$ is a real function describing the speed of the pedestrians. This function does not depend on the local density $\rho(t, x)$ but on the averaged density $\rho(t) * \eta(x)=\int_{\mathbb{R}^{N}} \rho(t, x-y) \eta(y) \mathrm{d} y$. The vector field, $\vec{\nu}(x)$ describes the direction that the pedestrian located in $x$ will follow, independently from the distribution of the pedestrians' density. Note that we are working here on the all of $\mathbb{R}^{N}$ and not on a subset of $\mathbb{R}^{N}$; thus, we are not working on a restriction to a room, for example. However, we can still introduce the presence of walls and obstacles in the choice of the vector field $\vec{\nu}$. Let us denote $\Omega \subset \mathbb{R}^{N}$ the space where the pedestrians are authorized to walk, e.g. a room. If we choose $\vec{\nu}(x)$ in a nice way (for example we can require that on the walls, i.e. for all $x \in \partial \Omega, \vec{\nu}(x)$ coincides with the entering normal to $\Omega$ ), then we can conclude to the invariance of the room. More precisely, if the initial density has support on some closed set $\Omega \subset \mathbb{R}^{N}$, then the solution will have support contained in $\Omega$ for all time. This remark allows us to avoid considering any boundaries and to have solutions on all $\mathbb{R}^{N}$.

Using the Kružkov theory on classical scalar conservation laws, we are able to prove: 
Theorem 2.1 (see $[7])$. Let $\rho_{0} \in\left(\mathbf{L}^{\mathbf{1}} \cap \mathbf{L}^{\infty} \cap \mathbf{B V}\right)\left(\mathbb{R}^{N}, \mathbb{R}_{+}\right)$. Assume $v \in\left(\mathscr{C}^{2} \cap \mathbf{W}^{2, \infty}\right)(\mathbb{R}, \mathbb{R}), \vec{\nu} \in\left(\mathscr{C}^{2} \cap\right.$ $\left.\mathbf{W}^{\mathbf{2}, \mathbf{1}}\right)\left(\mathbb{R}^{N}, \mathbb{R}^{N}\right), \eta \in\left(\mathscr{C}^{2} \cap \mathbf{W}^{\mathbf{2}, \infty}\right)\left(\mathbb{R}^{N}, \mathbb{R}\right)$. Then there exists a unique weak entropy solution $\rho=S_{t} \rho_{0} \in$ $\mathscr{C}^{0}\left(\mathbb{R}_{+}, \mathbf{L}^{\mathbf{1}}\left(\mathbb{R}^{N}, \mathbb{R}_{+}\right)\right)$to (1) with initial condition $\rho_{0}$. Furthermore we have the estimate

$$
\|\rho(t)\|_{\mathbf{L}^{\infty}} \leq\left\|\rho_{0}\right\|_{\mathbf{L}^{\infty}} e^{C t}
$$

where the constant $C$ depends on $v, \vec{\nu}$ and $\eta$.

For the definition of weak entropy solutions see Section 3; the proof is deferred to Section 3.2.1.

In Theorem 2.1, the hypotheses are very strong. Let us denote $\mathcal{P}\left(\mathbb{R}^{N}\right)$ the set of probability measures on $\mathbb{R}^{N}$ and $\mathcal{M}^{+}\left(\mathbb{R}^{N}\right)$ the set of positive measures on $\mathbb{R}^{N}$. In collaboration with G. Crippa, using now some tools from optimal transport theory and in particular tools on the continuity equation $\partial_{t} \rho+\operatorname{Div}(\rho b)=0$ (which is obtained from (1) when the nonlocal term is fixed, as in (2)), we obtained the better result:

Theorem 2.2 (see $[9])$. Let $\rho_{0} \in \mathcal{M}^{+}\left(\mathbb{R}^{N}\right)$. Assume $v \in\left(\mathbf{L}^{\infty} \cap \operatorname{Lip}\right)(\mathbb{R}, \mathbb{R}), \vec{\nu} \in\left(\mathbf{L}^{\infty} \cap \operatorname{Lip}\right)\left(\mathbb{R}^{N}, \mathbb{R}^{N}\right)$, $\eta \in\left(\mathbf{L}^{\infty} \cap \operatorname{Lip}\right)\left(\mathbb{R}^{N}, \mathbb{R}_{+}\right)$. Then there exists a unique weak measure solution $\rho \in \mathbf{L}^{\infty}\left(\mathbb{R}_{+}, \mathcal{M}^{+}\left(\mathbb{R}^{N}\right)\right)$ to (1) with initial condition $\rho_{0}$.

If furthermore $\rho_{0} \in \mathbf{L}^{\mathbf{1}}\left(\mathbb{R}^{N}, \mathbb{R}_{+}\right)$then for all $t \geq 0$, the solution $\rho$ satisfies also $\rho(t) \in \mathbf{L}^{\mathbf{1}}\left(\mathbb{R}^{N}, \mathbb{R}_{+}\right)$.

For the definition of weak measure solution see Section 4; the proof is deferred to Section 4.2.

Note that for the model (1), there is a priori no uniform $\mathbf{L}^{\infty}$ bound on the density. Indeed, heuristically, considering the case in which the density is maximal, equal to 1 , on the trajectory of a pedestrian located in $x$. If the averaged density around $x$ is strictly less than 1 (because, for example there is no one behind this pedestrian), then the speed $v(\rho * \eta)$ will be strictly positive, which means the pedestrian in $x$ will try to go forward, even though there is a queue in front of him. Consequently, we expect the density to become larger than one.

This behavior is not really unexpected in the case of panic. In fact, in some events the density attained up to 10 persons per square meter, which is obviously too much and a cause of deaths (see [13]). Consequently, it is quite satisfactory to recover this behavior. One of our goal in this context is then to introduce a cost functional allowing to characterize the cases in which the density is too high, and to find extrema of this functional. Let us introduce

$$
J_{T}\left(\rho_{0}\right)=\int_{0}^{T} \int_{\Omega} f\left(S_{t} \rho_{0}\right) \mathrm{d} x \mathrm{~d} t,
$$

where $\Omega \subset \mathbb{R}^{N}$ is the room, $\rho_{0}$ is the initial condition and $S_{t} \rho_{0}$ is the semi-group generated by Theorem 2.1 . We choose the function $f \in \mathscr{C}^{1}\left(\mathbb{R}, \mathbb{R}_{+}\right)$so that it is equal to zero for any density $\rho$ less than a fixed threshold $\rho_{c}$ and so that it is strictly increasing on $\left[\rho_{c},+\infty\left[\right.\right.$. Consequently, the functional $J_{T}$ above allows to characterize the solutions with too high density and in particular it vanishes if the set $\left\{(t, x) \in[0, T] \times \mathbb{R}^{N}: S_{t} \rho(x) \geq \rho_{c}\right\}$ has measure zero. We are then interested in finding the minima of this cost functional.

Using the Kružkov theory we prove the following differentiability result:

Theorem 2.3 (see $[6,7])$. Let $\rho_{0} \in\left(\mathbf{W}^{\mathbf{2}, \infty} \cap \mathbf{W}^{\mathbf{2}, \mathbf{1}}\right)\left(\mathbb{R}^{N}, \mathbb{R}_{+}\right), r_{0} \in\left(\mathbf{W}^{\mathbf{1}, \mathbf{1}} \cap \mathbf{L}^{\infty}\right)\left(\mathbb{R}^{N}, \mathbb{R}\right)$ and denote $\rho=S_{t} \rho_{0}$. Assume $v \in\left(\mathscr{C}^{4} \cap \mathbf{W}^{\mathbf{2}, \infty}\right)(\mathbb{R}, \mathbb{R}), \vec{\nu} \in\left(\mathscr{C}^{3} \cap \mathbf{W}^{\mathbf{2}, \mathbf{1}}\right)\left(\mathbb{R}^{N}, \mathbb{R}^{N}\right), \eta \in\left(\mathscr{C}^{3} \cap \mathbf{W}^{\mathbf{2}, \infty}\right)\left(\mathbb{R}^{N}, \mathbb{R}_{+}\right)$. Then there exists a unique weak entropy solution $r=\Sigma_{t}^{\rho} r_{0}$ to the Cauchy problem

$$
\partial_{t} r+\operatorname{Div}(r v(\rho * \eta) \vec{\nu}(x))=-\operatorname{Div}\left(\rho v^{\prime}(\rho * \eta) r * \eta \vec{\nu}(x)\right), \quad r(0)=r_{0} .
$$

Furthermore, the semi-group $S_{t}$ obtained in Theorem 2.1 is Gâteaux-differentiable, that is to say, for all $\rho_{0} \in\left(\mathbf{W}^{\mathbf{2}, \mathbf{1}} \cap \mathbf{W}^{\mathbf{2}, \infty}\right)\left(\mathbb{R}^{N}, \mathbb{R}_{+}\right), r_{0} \in\left(\mathbf{W}^{\mathbf{1}, \mathbf{1}} \cap \mathbf{L}^{\infty}\right)\left(\mathbb{R}^{N}, \mathbb{R}\right)$,

$$
\lim _{h \rightarrow 0}\left\|\frac{S_{t}\left(\rho_{0}+h r_{0}\right)-S_{t} \rho_{0}}{h}-\Sigma_{t}^{\rho} r_{0}\right\|_{\mathbf{L}^{1}}=0 .
$$


The proof is deferred to Section 3.2.2. It is not possible to obtain the same result by the use of optimal transport theory. Indeed, it seems already not possible to find a good definition of Gâteaux differentiability on the set of probability measures equipped with the Wasserstein distance of order 1.

Extrema of a cost functional. Once the differentiability result of Theorem 2.3 is available, a necessary condition of optimality is straightforward: we are now able to characterize the extrema of the cost functional $J_{T}$ given in (3) as stated below.

Proposition 2.4 (see Proposition 2.12 in $[6]$ ). Let $f \in \mathscr{C}^{1,1}\left(\mathbb{R} ; \mathbb{R}_{+}\right)$and $\psi \in \mathbf{L}^{\infty}\left(\mathbb{R}^{+} \times \mathbb{R}^{N} ; \mathbb{R}\right)$. Assume $S:[0, T] \times\left(\mathbf{L}^{\mathbf{1}} \cap \mathbf{L}^{\infty}\right)\left(\mathbb{R}^{N} ; \mathbb{R}\right) \rightarrow\left(\mathbf{L}^{\mathbf{1}} \cap \mathbf{L}^{\infty}\right)\left(\mathbb{R}^{N} ; \mathbb{R}\right)$ is $\mathbf{L}^{\mathbf{1}}$ Gâteaux differentiable.

If $\rho_{0} \in\left(\mathbf{L}^{\mathbf{1}} \cap \mathbf{L}^{\infty}\right)\left(\mathbb{R}^{N} ; \mathbb{R}\right)$ is solution of the problem: "Find $\min _{\rho_{0}} J_{T}\left(\rho_{0}\right)$ ", then, for all $r_{0} \in\left(\mathbf{L}^{\mathbf{1}} \cap\right.$ $\left.\mathbf{L}^{\infty}\right)\left(\mathbb{R}^{N} ; \mathbb{R}\right)$, we have: $\int_{0}^{T} \int_{\mathbb{R}^{N}} f^{\prime}\left(S_{t} \rho_{0}\right) \Sigma_{t}^{\rho_{0}} r_{0} \psi(t, x) \mathrm{d} x \mathrm{~d} t=0$.

\subsubsection{Orderly crowd}

In opposite to the previous model, for a model of orderly crowd it is required to have a uniform $\mathbf{L}^{\infty}$ bound on the density. In collaboration with R. M. Colombo and M. Garavello [5], we studied the equation

$$
\partial_{t} \rho+\operatorname{Div}\left[\rho v(\rho)\left(\vec{\nu}(x)-\frac{\nabla(\rho * \eta)}{\sqrt{1+\|\nabla(\rho * \eta)\|^{2}}}\right)\right]=0
$$

with $\rho_{0} \in\left(\mathbf{L}^{\mathbf{1}} \cap \mathbf{L}^{\infty} \cap \mathbf{B V}\right)\left(\mathbb{R}^{N} ; \mathbb{R}\right)$. In this model, the speed $v$ depends on the local density $\rho(t, x)$, which allows to prove some uniform bound in $\mathbf{L}^{\infty}$. The preferred direction of the pedestrians is still $\vec{\nu}(x)$, but they deviate from their optimal path trying to avoid entering regions with higher densities. Indeed, $(\rho * \eta)$ is an average of the crowd density around $x$ and $-\nabla(\rho * \eta)$ is a vector going in the direction opposite to the area of maximal averaged density. Due to the nonlinearity of the flow with respect to the local $\rho$, it is no longer possible to use optimal transport theory. Hence, we use Kružkov theory, to prove existence and uniqueness of solutions. We obtain the theorem:

Theorem 2.5 (see [5]). Assume that $v \in \mathscr{C}^{2}\left([0,1], \mathbb{R}_{+}\right)$satisfies $v(1)=0, \vec{\nu} \in\left(\mathscr{C}^{2} \cap \mathbf{W}^{\mathbf{2}, \mathbf{1}} \cap \mathbf{W}^{\mathbf{1}, \infty}\right)\left(\mathbb{R}^{N}, \mathbb{R}^{N}\right)$, and $\eta \in\left(\mathscr{C}^{3} \cap \mathbf{W}^{\mathbf{3}, \mathbf{1}} \cap \mathbf{W}^{\mathbf{2}, \infty}\right)\left(\mathbb{R}^{N}, \mathbb{R}\right)$. Then, for any $\rho_{0} \in\left(\mathbf{L}^{\mathbf{1}} \cap \mathbf{L}^{\infty} \cap \mathbf{B V}\right)\left(\mathbb{R}^{N},[0,1]\right)$, there exists a unique weak entropy solution $\rho \in \mathscr{C}^{0}\left(\mathbb{R}_{+}, \mathbf{L}^{\mathbf{1}}\left(\mathbb{R}^{N},[0,1]\right)\right)$ to $(5)$.

The proof of this theorem relies on Kružkov theory (see Section 3). For this model the density is uniformly bounded in $\mathbf{L}^{\infty}$, contrarily to the panic model, in which the $\mathbf{L}^{\infty}$ norm can grow exponentially in time.

Some difficulties now appear in proving that the pedestrians remain in the authorized area. Let us introduce the following invariance property:

(P): Let $\Omega \subset \mathbb{R}^{N}$ be region where the pedestrians are allowed to walk. The model (5) is invariant with respect to $\Omega$ if

$$
\operatorname{Supp}\left(\rho_{0}\right) \subset \Omega \quad \Rightarrow \quad \operatorname{Supp}(\rho(t)) \subset \Omega \quad \text { for all } t \geq 0 \text {. }
$$

To obtain that $(\mathbf{P})$ is satisfied, we have to require the preferred direction to be strongly entering the room (see [5, Proposition $3.1 \&$ Appendix A]).

Some interesting phenomena show up through numerical computations. First, when considering a crowd walking along a corridor, we observe the formation of lanes (see Figure 1).

Furthermore, this phenomenon seems very stable with respect to initial conditions and geometry. Indeed considering the room and initial distribution as in Figure 2, adding some various obstacles, we still have lanes, at least in large space (see Figures 3).

A further remarkable property of the model (5) is that it captures the following well-known, although sometimes counter intuitive phenomenon (Braess paradox): the evacuation time through an exit can be reduced 


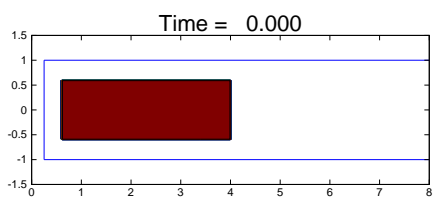

Time $=7.557$

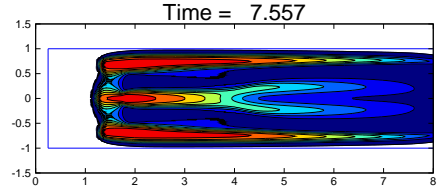

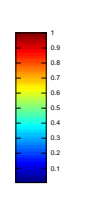

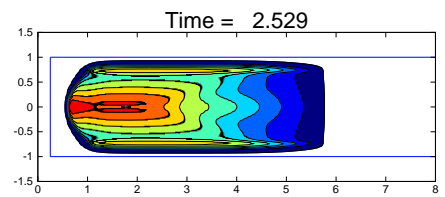

Time $=10.071$
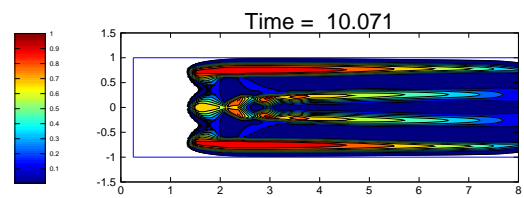
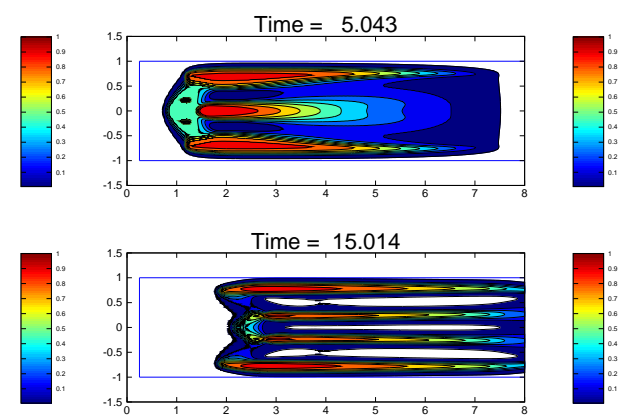

Figure 1. Solution to (5) at times $t=0,2.529,5.043,7.557,10.071,15.014$. First 3 lanes are formed, then the middle lane bifurcates forming the fourth lane. Picture from [5].

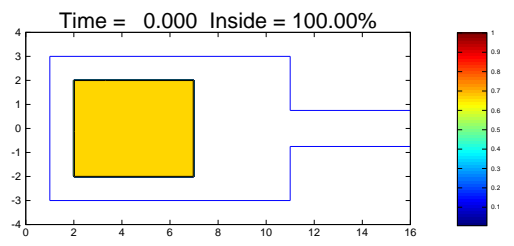

Figure 2. Initial datum and room geometry. Picture from [5].
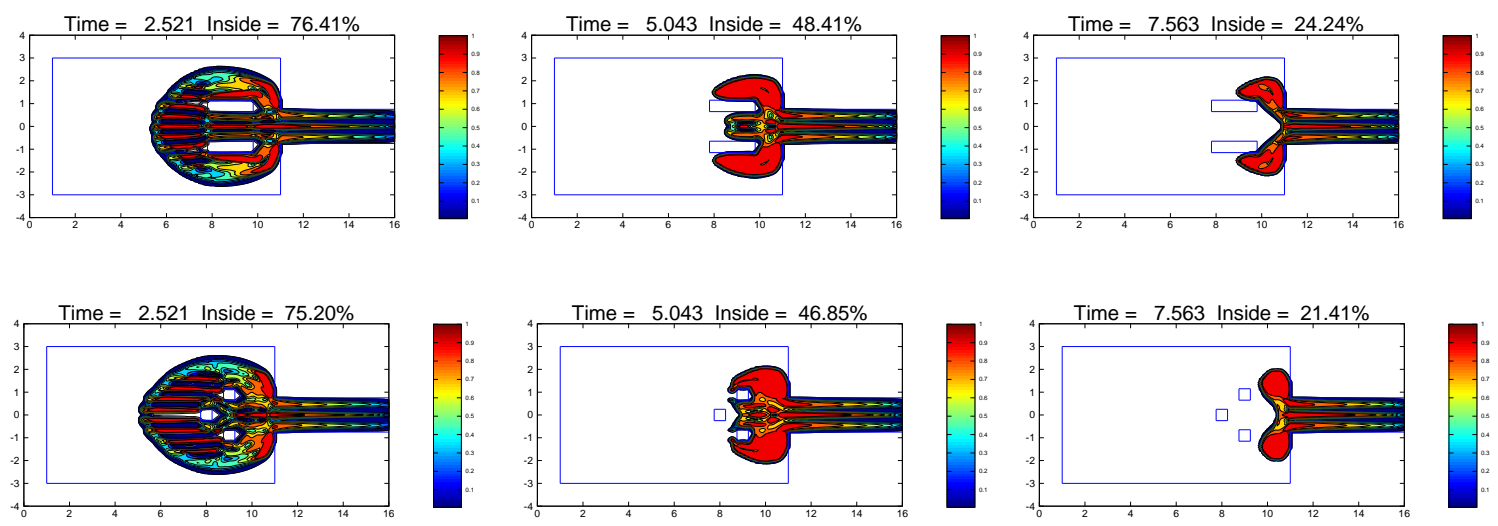

Figure 3. Solution to (5) with different geometries, computed at time $t=2.521,5.043$ and 7.563. Picture from [5].

by carefully positioning suitable "obstacles" that direct the outflow (see for instance [12] and the references therein). Indeed, looking at Figure 4, we observe that the time of exist with obstacles is slightly shorter than the one without any obstacle.

\subsection{Several populations}

A natural wish now is to extend the previous models to the case of several population with different objectives. 

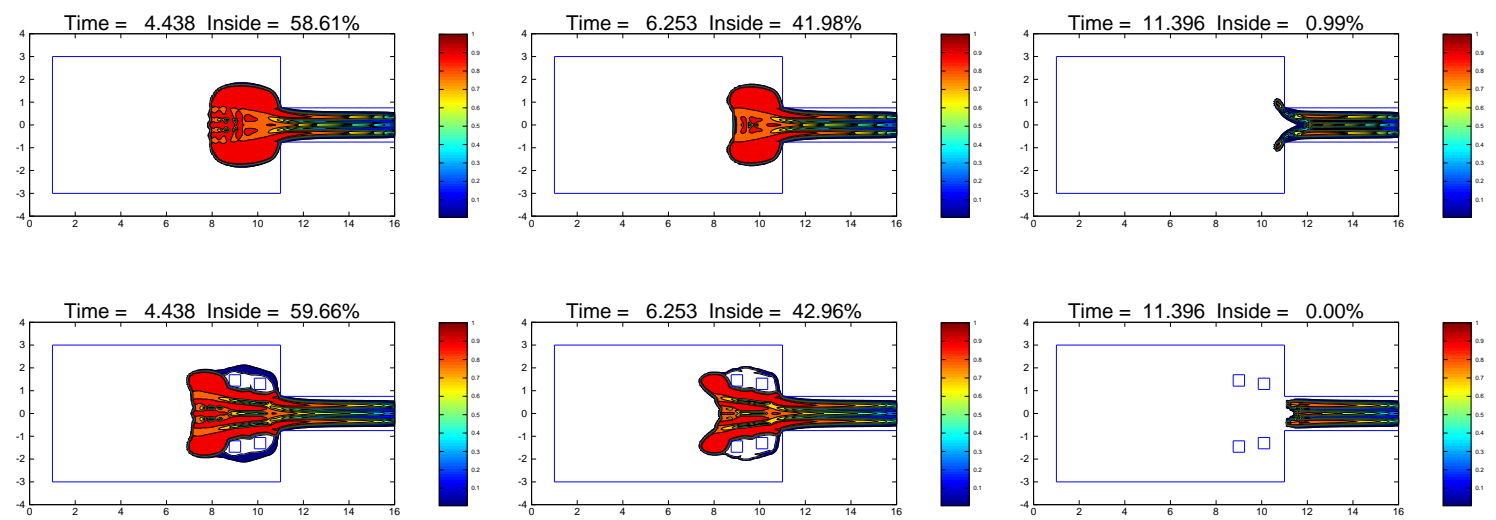

Figure 4. Solution to (5) with $\varepsilon=0.2$, at times $t=4.438,6.253,11.396$. On the first line, no obstacle is present. On the second line, 4 columns direct the crowd flow. The evacuation time in the latter case is shorter than in the former one. Picture from [5].

\subsubsection{Panic}

With several populations, we have to consider several densities and several equations. For two populations, extending the idea of the equation (1) to the case of several populations, we obtain the system

$$
\left\{\begin{array}{l}
\partial_{t} \rho_{1}+\operatorname{Div}\left(\rho_{1} v\left(\rho_{1} * \eta_{1}+\rho_{2} * \eta_{2}\right) \vec{\nu}_{1}(x)\right)=0 \\
\partial_{t} \rho_{2}+\operatorname{Div}\left(\rho_{2} v\left(\rho_{1} * \eta_{1}+\rho_{2} * \eta_{2}\right) \vec{\nu}_{2}(x)\right)=0
\end{array}\right.
$$

Here we consider that the speed $v$ depends on the average of the total density $\rho_{1}+\rho_{2}$. The difference of goals of the populations 1 and 2 is reflected in the choice of different preferred directions $\vec{\nu}_{1}$ and $\vec{\nu}_{2}$.

Note that the two equations in (6) are weakly coupled in the sense that the coupling appears only through the nonlocal term. Consequently, the definition of weak entropy solutions for this system has to be understood in the sense of Kružkov for each each equation and not in the sense of Lax for hyperbolic systems of conservation laws. We are able to prove theorems similar to the ones of section 2.1.1. In collaboration with R. M. Colombo [7], we obtained:

Theorem 2.6 (see $[7])$. Let $\rho_{0}=\left(\rho_{0,1}, \rho_{0,2}\right) \in\left(\mathbf{L}^{\mathbf{1}} \cap \mathbf{L}^{\infty} \cap \mathbf{B V}\right)\left(\mathbb{R}^{N}, \mathbb{R}_{+}^{2}\right)$. Assume that, for $i \in\{1,2\}$, $v_{i} \in\left(\mathscr{C}^{2} \cap \mathbf{W}^{2, \infty}\right)(\mathbb{R}, \mathbb{R}), \vec{\nu}_{i} \in\left(\mathscr{C}^{2} \cap \mathbf{W}^{\mathbf{2}, \mathbf{1}}\right)\left(\mathbb{R}^{N}, \mathbb{R}^{N}\right), \eta_{i} \in\left(\mathscr{C}^{2} \cap \mathbf{W}^{\mathbf{2}, \infty}\right)\left(\mathbb{R}^{N}, \mathbb{R}\right)$. Then there exists a unique weak entropy solution $\rho=S_{t} \rho_{0} \in \mathscr{C}^{0}\left(\mathbb{R}_{+}, \mathbf{L}^{\mathbf{1}}\left(\mathbb{R}^{N}, \mathbb{R}_{+}^{2}\right)\right)$ to (6) with initial condition $\rho_{0}$. Furthermore we have the estimate

where the constant $C$ depends on $v, \vec{\nu}$ and $\eta$.

$$
\|\rho(t)\|_{\mathbf{L}^{\infty}} \leq\left\|\rho_{0}\right\|_{\mathbf{L}^{\infty}} e^{C t}
$$

Similarly as for one population, it is possible with several populations to prove the Gâteaux-differentiability thanks to Kružkov theory (see [7, Theorem 2.2]).

As in Theorem 2.6, the hypotheses of Theorem 2.6 are very strong. In collaboration with G. Crippa [9], using tools from optimal transport theory and properties of the continuity equations, we obtained the better result:

Theorem 2.7 (see $[9])$. Let $\rho_{0} \in \mathcal{P}\left(\mathbb{R}^{N}\right)^{2}$. Assume $v_{i} \in\left(\mathbf{L}^{\infty} \cap \operatorname{Lip}\right)(\mathbb{R}, \mathbb{R}), \vec{\nu}_{i} \in\left(\mathbf{L}^{\infty} \cap \operatorname{Lip}\right)\left(\mathbb{R}^{N}, \mathbb{R}^{N}\right), \eta_{i} \in$ $\left(\mathbf{L}^{\infty} \cap \operatorname{Lip}\right)\left(\mathbb{R}^{N}, \mathbb{R}_{+}\right)$. Then there exists a unique weak measure solution $\rho \in \mathbf{L}^{\infty}\left(\mathbb{R}_{+}, \mathcal{P}\left(\mathbb{R}^{N}\right)^{2}\right)$ to (6) with initial condition $\rho_{0}$.

If furthermore $\rho_{0} \in \mathbf{L}^{\mathbf{1}}\left(\mathbb{R}^{N}, \mathbb{R}_{+}\right)$then for all $t \geq 0$, we have $\rho(t) \in \mathbf{L}^{\mathbf{1}}\left(\mathbb{R}^{N}, \mathbb{R}_{+}\right)$.

The idea of the proof for this theorem is given in Section 4.

Interaction continuum / individuals. Note that in the framework of Theorem 2.7, we are dealing with measure solutions. This context allows us to describe a coupling between a group of density $\rho_{1}$ and an individual 
located in $p(t)$. Indeed, let us assume that $\rho_{1} \in \mathbf{L}^{\infty}\left(\mathbb{R}_{+}, \mathbf{L}^{\mathbf{1}}\left(\mathbb{R}^{N}, \mathbb{R}_{+}\right)\right)$and $\rho_{2}=\delta_{p(t)}$ is a Dirac measure. Then we have the following ODE/PDE coupling:

$$
\left\{\begin{array}{l}
\partial_{t} \rho_{1}+\operatorname{div}\left(\rho_{1} v\left(\rho_{1} * \eta_{1}(x)+\eta_{2}(x-p(t))\right) \vec{\nu}_{1}(x)\right)=0 \\
\dot{p}(t)=v\left(\rho_{1} * \eta_{1}(p(t))+\eta_{2}(0)\right) \vec{\nu}_{2}(p(t)) .
\end{array}\right.
$$

\subsubsection{Orderly crowd}

We now extend (5) to the case of several populations. We consider here not only that $\rho_{1}$ and $\rho_{2}$ have two different goals, but also that $\rho_{1}$ is repelled by $\rho_{2}$, and that $\rho_{2}$ is repelled by $\rho_{1}$. Let us denote $\varepsilon_{i}$ the parameter of self-interaction and $\varepsilon_{o}$ the parameter of interaction with the other population. We obtain:

$$
\left\{\begin{array}{c}
\partial_{t} \rho_{1}+\operatorname{Div}\left(\rho_{1} v_{1}\left(\rho_{1}\right)\left(\vec{\nu}_{1}(x)-\varepsilon_{i} \frac{\nabla \rho_{1} * \eta_{1}}{\sqrt{1+\left\|\nabla \rho_{1} * \eta_{1}\right\|^{2}}}-\varepsilon_{o} \frac{\nabla \rho_{2} * \eta_{2}}{\sqrt{1+\left\|\nabla \rho_{2} * \eta_{2}\right\|^{2}}}\right)\right)=0, \\
\partial_{t} \rho_{2}+\operatorname{Div}\left(\rho_{2} v_{2}\left(\rho_{2}\right)\left(\vec{\nu}_{2}(x)-\varepsilon_{o} \frac{\nabla \rho_{1} * \eta_{1}}{\sqrt{1+\left\|\nabla \rho_{1} * \eta_{1}\right\|^{2}}}-\varepsilon_{i} \frac{\nabla \rho_{2} * \eta_{2}}{\sqrt{1+\left\|\nabla \rho_{2} * \eta_{2}\right\|^{2}}}\right)\right)=0 .
\end{array}\right.
$$

Remark 2.8. Note that the interaction between the equations is only in the nonlocal term.

We obtain again existence and uniqueness of weak entropy solutions thanks to Kružkov theory (see [7, Theorem 3.2]). The obtained theorem is similar to Theorem 2.5 so we don't rewrite it.

A classical situation considered in the engineering literature is that of two groups of people moving in opposite directions and crossing each other. The numerical integration (see Figure 5), shows formation of lanes that are not superimposing as described in the engineering literature ( $[10,14]$ or $[1])$.

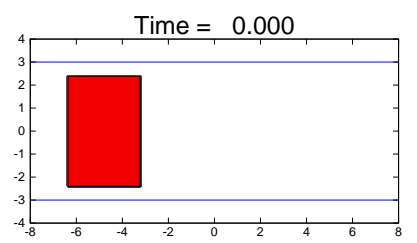

Time $=0.000$

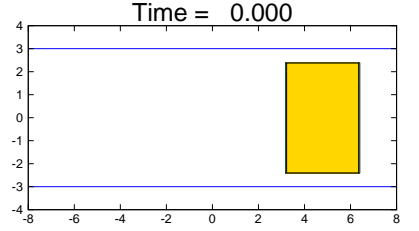

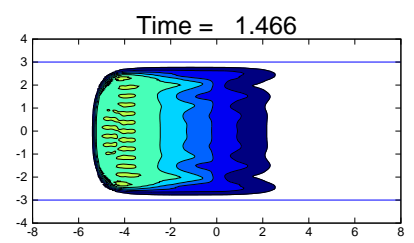

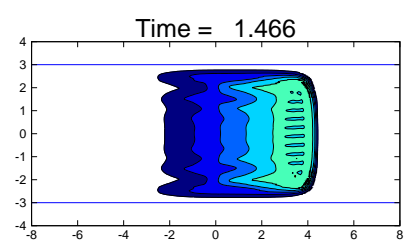

Time $=1.971$

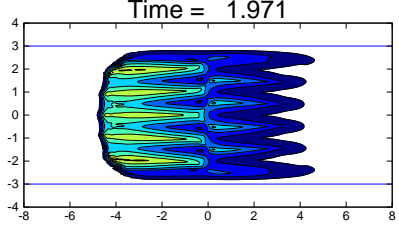

Time $=1.971$

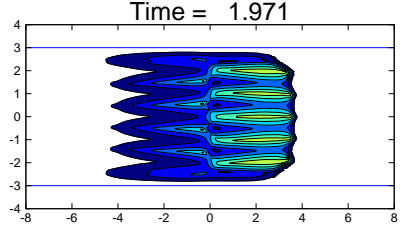

Time $=2.981$

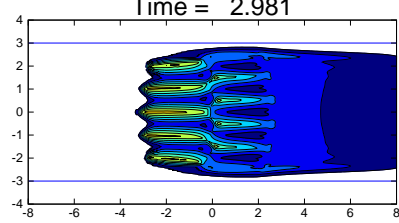

Time $=2.981$

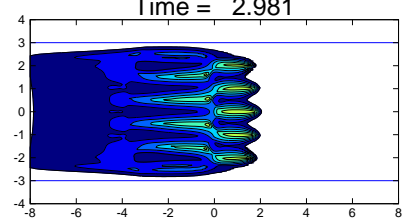

Figure 5. Numerical integration of (8). Above, the population $\rho_{1}$ moving to the right and, below, $\rho_{2}$ moving to the left. Note the lanes that are formed. First, due to the self-interaction $\left(\varepsilon_{i}=0.3\right)$ within each populations and then due to the crossing between the two populations $\left(\varepsilon_{o}=0.7\right)$. The latter lanes of different populations do not superimpose. Picture from [7].

Another situation developing interesting features is the following: two populations are initially uniformly distributed in the same region. At time $t=0$, the first populations starts moving towards an exit (first line of Figure 6), on the right while the second moves only to let the first one pass (second line of Figure 6).

\subsubsection{Interaction group / isolated agent}

Let us finally introduce a model that would take into account the situation in which an isolated individual interact with a crowd. We think for example to the situation in which a predator is running after a group of 

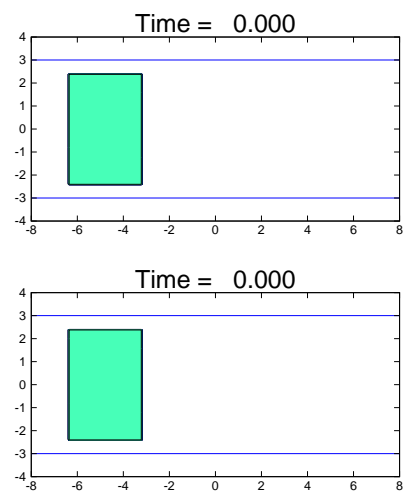

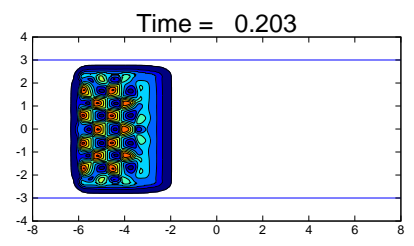

Time $=0.203$

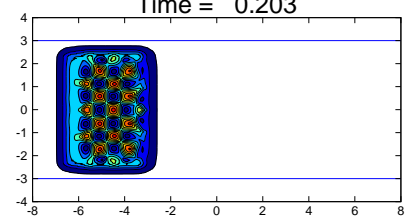

Time $=1.213$

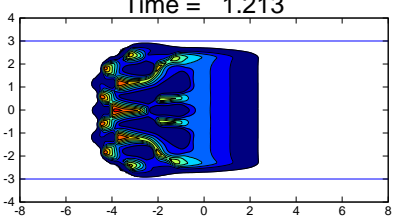

Time $=1.213$

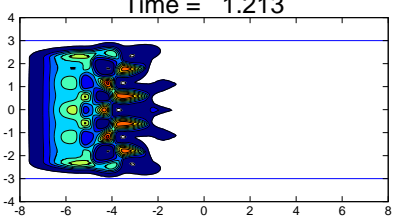

Time $=2.476$

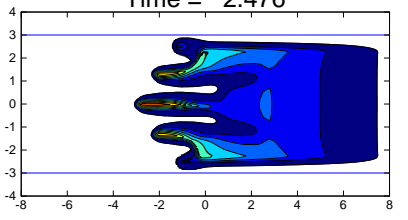

Time $=2.476$

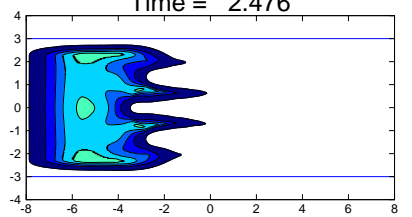

Figure 6. Numerical integration of (8) when with $\varepsilon_{i}=0, \varepsilon_{o}=0.3$. Above, the population $\rho_{1}$ and, below, $\rho_{2}$. Note first the formation of small clusters separating the two populations, then lanes and, finally, a sort of fingering. Picture from [7].

preys. We can also think to the case of a leader willing to carry a group of followers to a given region. Let $\rho \in \mathbb{R}_{+}$be the density of the group and $p \in \mathbb{R}^{k}$ be the position of an isolated agent (e.g. a leader or a predator). We describe the interaction by the coupling (see [4]):

$$
\left\{\begin{array}{l}
\partial_{t} \rho+\operatorname{Div}(\rho V(t, x, \rho, p(t)))=0, \\
\dot{p}=\varphi(t, p,(A \rho(t))(p(t))),
\end{array} \quad(t, x) \in \mathbb{R}^{+} \times \mathbb{R}^{N},\right.
$$

with initial conditions $\rho(0, x)=\rho_{0}(x), p(0)=p_{0}$.

Using once again Kružkov theory and tools on the stability of ordinary differential equations, in collaboration with R. M. Colombo, we proved existence and uniqueness of solutions (see [4, Theorem 2.2]).

For example, we can consider:

- Followers / Leader:

$$
\left\{\begin{array}{l}
\partial_{t} \rho+\operatorname{Div}\left(\rho v(\rho)(p(t)-x) e^{-\|p-x\|}\right)=0 \\
\dot{p}=(1+\rho * \eta(p(t))) \vec{\psi}(t)
\end{array}\right.
$$

Here the vector $p \in \mathbb{R}^{2}$ is the position of the leader and $\rho$ is the density of the group of followers. The function $v(\rho)$ describes essentially the speed of the followers and is, as usual, a decreasing function, vanishing in $\rho=1$; the direction of a follower located in $x$ is given by the vector $p(t)-x$, directed toward the leader. The velocity of the leader is increasing with respect to the averaged density $\rho * \eta$, computed in the position of the leader. Indeed, we expect the leader to wait for the followers to join him when the density of followers is small around him, and to accelerate when the density of followers becomes bigger; this means low speed when the averaged density is small and high speed when the averaged density is maximal. The direction of the leader is a chosen vector field $\vec{\psi}(t)$.

- Sheep / Dogs:

$$
\left\{\begin{array}{l}
\partial_{t} \rho+\operatorname{Div}\left(\rho v(\rho)\left(\vec{\nu}(x)+\sum_{i=1}^{n}\left(x-p_{i}\right) e^{-\left\|p_{i}-x\right\|}\right)\right)=0 \\
\dot{p}_{i}=\frac{(\rho * \nabla \eta)^{\perp}\left(p_{i}(t)\right)}{\sqrt{1+\left\|\rho * \nabla \eta\left(p_{i}(t)\right)\right\|^{2}}}, \quad \text { for all } i \in\{1, \ldots n\}
\end{array}\right.
$$


In this example, there are $n$ dogs, located in $p_{i}(t) \in \mathbb{R}^{2}$ for $i \in\{1, \ldots, n\}$ and a group of sheep of density $\rho(t, x)$. The dogs want to constrain the sheep to a given area by running around them and the sheep are afraid by the dogs. As above, the speed of the sheep is given by the decreasing function $v(\rho)$ that vanishes in $\rho=1$. The direction of a sheep located in $x$ is a sum of two terms. The first one is their preferred direction $\vec{\nu}(x)$; the second one is given by the vector $\sum_{i=1}^{n}\left(x-p_{i}(t)\right) e^{-\left\|p_{i}-x\right\|}$ representing the repulsive effects of the dogs on the sheep, and its presence is is less felt if it is too far away. Each dog run around the flock tacking the direction perpendicular to the direction of maximal averaged density.

- Preys / Predator (see Figure 7):

$$
\left\{\begin{array}{l}
\partial_{t} \rho+\operatorname{Div}\left(\rho v(\rho)\left(1+e^{-\|x-p(t)\|}(x-p(t))\right)\right)=0 \\
\frac{\mathrm{d}^{2} p}{\mathrm{~d} t^{2}}=(\rho * \nabla) \eta(p(t))
\end{array}\right.
$$

Here a predator, located in $p(t) \in \mathbb{R}^{3}$ is pursuing a group of preys of density $\rho(t, x)$. For example, we can think to an hawk pursuing a swarm of little birds or to a shark pursuing a shoal of little fishes. As above, the speed of the preys is given by the decreasing function $v(\rho)$ that vanishes in $\rho=1$. The direction of a prey located in $x$ is given by the vector $(x-p(t)) e^{-\|p(t)-x\|}$ so that the predator has a repulsive effect on the preys, and its presence is less felt if it is too far away. The acceleration of the predator is directed toward the maximal averaged density of preys, as felt from its position, so it is directed as $\nabla(\rho * \eta)(p(t))$.
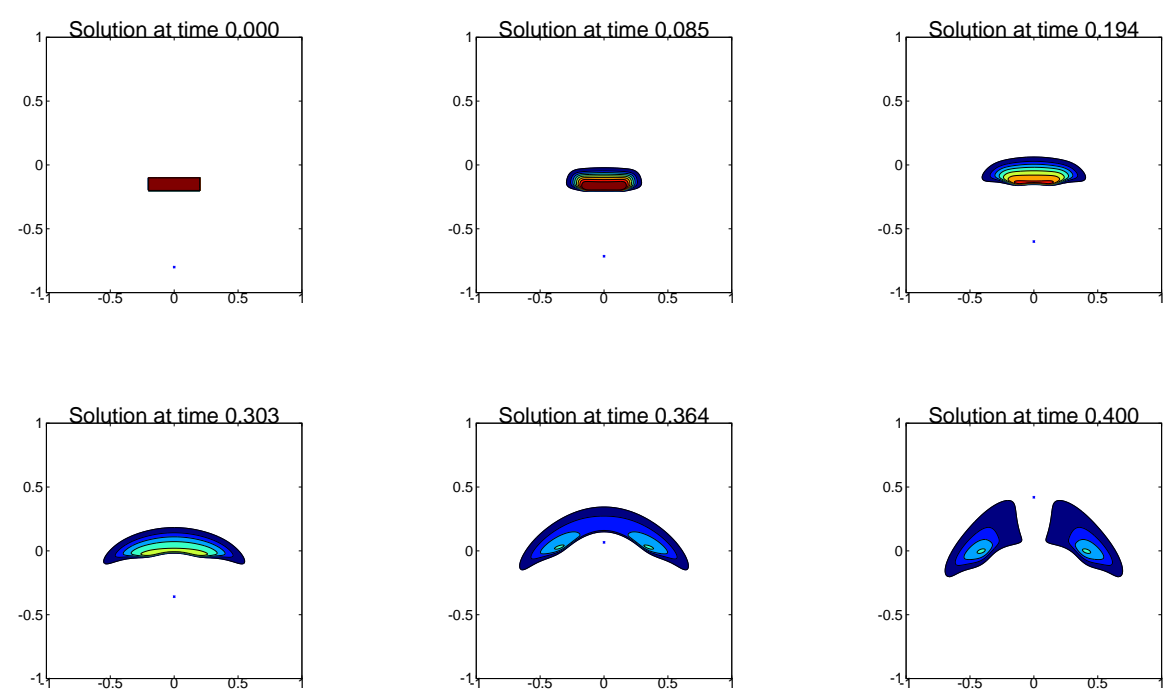

FiguRE 7. Numerical solution of (10) in a predator/prey interaction. Picture from [4].

Note that, in the framework of Theorem 2.7, in (7) we also obtained a coupling PDE/ODE. However, in Theorem 2.7, there are restrictions on the flow as we can only deal with continuity equations, once the nonlocal term is fixed. Contrarily, it is possible to have a flow non-linear in $\rho$ in (9). The interest of this is that, with a well-chosen nonlinearity with respect to $\rho$ in the first equation of (9), we are able to provide for (9) an a priori uniform bound on the $\mathbf{L}^{\infty}$ norm (which can not be proved for (7)): for instance, in the three examples above, if $v(1)=0$, then $0 \leq \rho_{0} \leq 1$ implies $0 \leq \rho(t) \leq 1$ for all $t \geq 0$. 


\section{USING KRUŽKOV THEORY}

\subsection{General Theory on scalar conservation laws}

In this section, we consider classical scalar conservation laws.

$$
\partial_{t} u+\operatorname{Div} f(t, x, u)=F(t, x, u), \quad u(0, x)=u_{0}(x),
$$

where $f$ is the flow and $F$ is the source. Note that, Div stands for the total divergence whereas div stands for the partial divergence. Thus $\operatorname{Div} f(t, x, u(t, x))=\operatorname{div} f(t, x, u(t, x))+\partial_{u} f(t, x, u(t, x)) \cdot \nabla u(t, x)$.

Let us recall the definition of weak entropy solution:

Definition 3.1. The function $\rho \in \mathbf{L}^{\infty}\left([0, T] \times \mathbb{R}^{N}, \mathbb{R}\right)$ is called weak entropy solution to the Cauchy problem (11) if it satisfies for all $k \in \mathbb{R}$ and any test-function $\varphi \in \mathscr{C}_{c}^{0}(]-\infty, T\left[\times \mathbb{R}^{N}, \mathbb{R}_{+}\right)$,

$$
\begin{array}{r}
\int_{\mathbb{R}_{+}^{*}} \int_{\mathbb{R}^{N}}\left[(u-k) \partial_{t} \varphi+(f(t, x, u)-f(t, x, k)) \nabla_{x} \varphi+(F(t, x, u)-\right. \\
\operatorname{div} f(t, x, k)) \varphi] \operatorname{sign}(u-k) \mathrm{d} x \mathrm{~d} t \\
+\int_{\mathbb{R}^{N}}\left|\rho_{0}(x)-k\right| \varphi(0, x) \mathrm{d} x \geq 0 .
\end{array}
$$

We also recall the well-known Kružkov theorem [17, Theorem 4], giving existence and uniqueness of weak entropy solutions.

Theorem 3.2 (Kružkov). Let $u_{0} \in\left(\mathbf{L}^{\infty} \cap \mathbf{L}^{\mathbf{1}}\right)\left(\mathbb{R}^{N}, \mathbb{R}\right)$. For all $A, T \geq 0$, we denote $\Omega_{T}^{A}=[0, T] \times \mathbb{R}^{N} \times[-A, A]$ and $\Omega=\mathbb{R}_{+} \times \mathbb{R}^{N} \times \mathbb{R}$. Under the conditions $f \in \mathscr{C}^{0}\left(\Omega ; \mathbb{R}^{N}\right), F \in \mathscr{C}^{0}(\Omega ; \mathbb{R})$ and

$(\mathbf{K})\left\{\begin{array}{l}f \in \mathscr{C}^{0}\left(\Omega ; \mathbb{R}^{N}\right), \quad F \in \mathscr{C}^{0}(\Omega ; \mathbb{R}), \text { and for all } T, A>0: \\ f, F \text { have continuous derivatives: } \partial_{u} f, \partial_{u} \nabla f, \nabla^{2} f, \partial_{u} F, \nabla F, \\ \text { for all } T, A>0, \quad \partial_{u} f \in \mathbf{L}^{\infty}\left(\Omega_{T}^{A}\right), \quad F-\operatorname{div} f \in \mathbf{L}^{\infty}\left(\Omega_{T}^{A}\right), \quad \partial_{u}(F-\operatorname{div} f) \in \mathbf{L}^{\infty}\left(\Omega_{T}^{A}\right)\end{array}\right.$

there exists a unique weak entropy solution $u \in \mathbf{L}^{\infty}\left([0, T] ; \mathbf{L}^{\mathbf{1}}\left(\mathbb{R}^{N} ; \mathbb{R}\right)\right)$ of (11) that is right-continuous in time.

Let $v_{0} \in\left(\mathbf{L}^{\mathbf{1}} \cap \mathbf{L}^{\infty}\right)\left(\mathbb{R}^{N} ; \mathbb{R}\right)$. Let $u$ be the solution associated to the initial condition $u_{0}$ and $v$ be the solution associated to the initial condition $v_{0}$. Let $M$ be such that $M \geq \sup \left(\|u\|_{\mathbf{L}^{\infty}\left(\mathbb{R}_{+} \times \mathbb{R}^{N} ; \mathbb{R}\right)},\|v\|_{\mathbf{L}^{\infty}\left([0, T] \times \mathbb{R}^{N} ; \mathbb{R}\right)}\right)$. Then, for all $t \in[0, T]$, with $\gamma=\left\|\partial_{u} F\right\|_{\mathbf{L}^{\infty}\left(\Omega_{T}^{M}\right)}$, we have

$$
\|(u-v)(t)\|_{\mathbf{L}^{\mathbf{1}}} \leq e^{\gamma t}\left\|u_{0}-v_{0}\right\|_{\mathbf{L}^{1}} .
$$

3.1.1. Estimate on the dependence with respect to flow and source

Let us now consider a pair of different flows $f, g \in \mathscr{C}^{2}\left([0, T] \times \mathbb{R}^{N} \times \mathbb{R} ; \mathbb{R}^{N}\right)$ and different sources $F, G \in$ $\mathscr{C}^{1}\left([0, T] \times \mathbb{R}^{N} \times \mathbb{R} ; \mathbb{R}\right)$, let us denote $u, v$ the solutions of

$$
\begin{aligned}
\partial_{t} u+\operatorname{Div} f(t, x, u) & =F(t, x, u), & u(0, \cdot) & =u_{0}, \\
\partial_{t} v+\operatorname{Div} g(t, x, v) & =G(t, x, v), & & v(0, \cdot)=v_{0},
\end{aligned}
$$

with initial conditions $u_{0}, v_{0} \in\left(\mathbf{L}^{\mathbf{1}} \cap \mathbf{L}^{\infty} \cap \mathbf{B V}\right)\left(\mathbb{R}^{N}, \mathbb{R}\right)$. We want here to estimate $\|(u-v)(t)\|_{\mathbf{L}^{1}}$ with $f-g$, $F-G, u_{0}-v_{0}$. To do that, we use the doubling variable method due to Kružkov [17]. This strategy was already employed by Lucier [19] and Bouchut \& Perthame [3] to study the case in which the flows $f, g$ depend only on $u$ and the source are identically zero $(F=G=0)$. A key ingredient in the previous results is that the solution of $\partial_{t} u+\operatorname{Div}(f(u))=0$ with initial condition $u_{0} \in\left(\mathbf{L}^{\infty} \cap \mathbf{L}^{\mathbf{1}} \cap \mathbf{B V}\right)\left(\mathbb{R}^{N}, \mathbb{R}\right)$ satisfies $\operatorname{TV}(u(t)) \leq \operatorname{TV}\left(u_{0}\right)$.

Let us remind the definition of total variation. 
Definition 3.3. For $u \in \mathbf{L}_{\text {loc }}^{1}\left(\mathbb{R}^{N} ; \mathbb{R}\right)$ we denote the total variation of $u$ :

$$
\operatorname{TV}(u)=\sup \left\{\int_{\mathbb{R}^{N}} u \operatorname{div} \Psi ; \quad \Psi \in \mathscr{C}_{c}^{1}\left(\mathbb{R}^{N} ; \mathbb{R}^{N}\right), \quad\|\Psi\|_{\mathbf{L}^{\infty}} \leq 1\right\} .
$$

The space of function with bounded variation is then defined as $\mathbf{B V}\left(\mathbb{R}^{N} ; \mathbb{R}\right)=\left\{u \in \mathbf{L}_{\text {loc }}^{\mathbf{1}} ; \operatorname{TV}(u)<\infty\right\}$.

Remark 3.4. If $u \in\left(\mathscr{C}^{1} \cap \mathbf{W}^{\mathbf{1}, \mathbf{1}}\right)\left(\mathbb{R}^{N}, \mathbb{R}\right)$ then $\operatorname{TV}(u)=\|\nabla u\|_{\mathbf{L}^{\mathbf{1}}}$. When $f$ and $F$ are not depending on $u$, we have

$$
u_{0} \in\left(\mathbf{L}^{\infty} \cap \mathbf{B V}\right)\left(\mathbb{R}^{N}, \mathbb{R}\right) \Rightarrow \forall t \geq 0, \quad u(t) \in\left(\mathbf{L}^{\infty} \cap \mathbf{B V}\right)\left(\mathbb{R}^{N}, \mathbb{R}\right)
$$

and, with $\gamma=\left\|\partial_{u} F\right\|_{\mathbf{L}^{\infty}\left(\Omega_{T}^{M}\right)}$, we have the estimate: $\operatorname{TV}(u(t)) \leq \operatorname{TV}\left(u_{0}\right) e^{\gamma t}$.

Let us remind the result from Lucier:

Theorem 3.5 (see [19]). Let $f, g: \mathbb{R} \rightarrow \mathbb{R}^{N}$ be globally Lipschitz, let $u_{0}, v_{0} \in\left(\mathbf{L}^{\mathbf{1}} \cap \mathbf{L}^{\infty}\right)\left(\mathbb{R}^{N} ; \mathbb{R}\right)$ be initial conditions of

$$
\partial_{t} u+\operatorname{Div} f(u)=0, \quad \partial_{t} v+\operatorname{Div} g(v)=0 .
$$

Assume furthermore that $v_{0} \in \mathbf{B V}\left(\mathbb{R}^{N} ; \mathbb{R}\right)$. Then for all $t \geq 0$,

$$
\|(u-v)(t)\|_{\mathbf{L}^{1}} \leq\left\|u_{0}-v_{0}\right\|_{\mathbf{L}^{1}}+C t \operatorname{TV}\left(v_{0}\right) \mathbf{L i p}(f-g)
$$

where $C>0$ is a given constant.

We want to generalize this theorem. Let us introduce the set of hypotheses:

$(\mathbf{F S}):$ for all $U, T>0, \quad \partial_{u} F \in \mathbf{L}^{\infty}\left(\Omega_{T}^{U}\right), \quad \int_{0}^{T} \int_{\mathbb{R}^{N}}\|(F-\operatorname{div} f)(t, x, \cdot)\|_{\mathbf{L}^{\infty}([-U, U] ; \mathbb{R})} \mathrm{d} x \mathrm{~d} t<+\infty$

Denoting $\Omega_{T}^{V}=[0, T] \times \mathbb{R}^{N} \times[-V, V]$, we obtain:

Theorem 3.6 (see $[18])$. Assume that $(f, F),(g, G)$ satisfy $(\boldsymbol{K})$, that $(f-g, F-G)$ satisfies $(\boldsymbol{F S})$. Let $u_{0}, v_{0} \in$ $\left(\mathbf{L}^{1} \cap \mathbf{L}^{\infty} \cap \mathbf{B V}\right)\left(\mathbb{R}^{N} ; \mathbb{R}\right)$. Let $u$ and $v$ be the solutions of $(13)$ and (14) associated to $(f, F)$ and $(g, G)$ with initial conditions $u_{0}$ and $v_{0}$. Assume furthermore that $\mathrm{TV}(u(t))<\infty$ for all $t \geq 0$.

Let $V=\max \left(\|u\|_{\mathbf{L}^{\infty}},\|v\|_{\left.\mathbf{L}^{\infty}\right)}\right)$ and $\kappa=\left\|\partial_{u} F\right\|_{\mathbf{L}^{\infty}\left(\Omega_{T}^{V}\right)}$. Then for all $t \in[0, T]$ :

$$
\begin{aligned}
\|(u-v)(t)\|_{\mathbf{L}^{1}} \leq & e^{\kappa t}\left\|u_{0}-v_{0}\right\|_{\mathbf{L}^{1}}+e^{\kappa t} \sup _{\tau \in[0, t]}(\operatorname{TV}(u(\tau))) \int_{0}^{t}\left\|\partial_{u}(f-g)(\tau)\right\|_{\mathbf{L}^{\infty}\left(\mathbb{R}^{N} \times[-V ; V]\right)} \mathrm{d} \tau \\
& +\int_{0}^{t} e^{\kappa(t-\tau)} \int_{\mathbb{R}^{N}}\|((F-G)-\operatorname{div}(f-g))(\tau, x, \cdot)\|_{\mathbf{L}^{\infty}([-V, V])} \mathrm{d} x \mathrm{~d} \tau
\end{aligned}
$$

The proof of this theorem is based on the Kružkov doubling variables method and is detailed in [18]. In some particular cases, we recover known estimates:

- If $f=g$ and $F=G$, then $\kappa=\gamma$ and we recover (12).

- If $f(u), g(u), F=G=0: \kappa=0$ and we recover the result of Lucier (see Theorem 3.5)

$$
\|(u-v)(t)\|_{\mathbf{L}^{1}} \leq\left\|u_{0}-v_{0}\right\|_{\mathbf{L}^{1}}+t \operatorname{TV}\left(u_{0}\right)\left\|\partial_{u}(f-g)\right\|_{\mathbf{L}^{\infty}\left(\Omega_{T}^{V}\right)} .
$$

- If $f(t, x), F(t, x)$, then $\kappa=0, u(t, x)=u_{0}(x)+\int_{0}^{t}(F-\operatorname{div} f)(\tau, x) \mathrm{d} \tau$, and

$$
\|(u-v)(t)\|_{\mathbf{L}^{1}} \leq\left\|u_{0}-v_{0}\right\|_{\mathbf{L}^{1}}+\int_{0}^{t} \int_{\mathbb{R}^{N}}|((F-G)-\operatorname{div}(f-g))(\tau, x)| \mathrm{d} x \mathrm{~d} \tau .
$$




\subsubsection{Total variation estimate}

To complete Theorem 3.6, we need an estimate on the total variation, defined in Definition 3.3. To do so we need a few more hypotheses. Indeed, there is no reason why the total variation should be bounded for all time: for instance, let us consider the equation $\partial_{t} u+\partial_{x} \cos x=0, u_{0}=0$. The solution of this Cauchy problem is $u(t, x)=t \sin x$ whose total variation is 0 at time 0 and $+\infty$ for any time $t>0$.

Our goal now is to give a general estimate on the total variation. Let us introduce the following set of hypotheses

$$
(\mathbf{T V}):\left\{\begin{array}{l}
\text { for all } A, T>0, \quad \nabla \partial_{u} f \in \mathbf{L}^{\infty}\left(\Omega_{T}^{A} ; \mathbb{R}^{N \times N}\right), \quad \partial_{u} F \in \mathbf{L}^{\infty}\left(\Omega_{T}^{A} ; \mathbb{R}\right) \\
\int_{0}^{T} \int_{\mathbb{R}^{N}}\|\nabla(F-\operatorname{div} f)(t, x, \cdot)\|_{\mathbf{L}^{\infty}\left([-A, A] ; \mathbb{R}^{N}\right)} \mathrm{d} x \mathrm{~d} t<\infty
\end{array}\right.
$$

We obtain

Theorem 3.7. Let us assume $(f, F)$ satisfies $(\boldsymbol{K})$ - $(\boldsymbol{T} \boldsymbol{V})$. Denote $W_{N}=\int_{0}^{\pi / 2}(\cos \theta)^{N} \mathrm{~d} \theta, M=\|u\|_{\mathbf{L}^{\infty}\left([0, T] \times \mathbb{R}^{N}\right)}$, and

$$
\kappa_{0}=(2 N+1)\left\|\nabla \partial_{u} f\right\|_{\mathbf{L}^{\infty}\left(\Omega_{T}^{M}\right)}+\left\|\partial_{u} F\right\|_{\mathbf{L}^{\infty}\left(\Omega_{T}^{M}\right)} .
$$

Then, the weak entropy solution $u$ of $(11)$ satisfies $u(t) \in \mathbf{B V}\left(\mathbb{R}^{N} ; \mathbb{R}\right)$ for all $t>0$, and

$$
\operatorname{TV}(u(T)) \leq \operatorname{TV}\left(u_{0}\right) e^{\kappa_{0} T}+N W_{N} \int_{0}^{T} e^{\kappa_{0}(T-t)} \int_{\mathbb{R}^{N}}\|\nabla(F-\operatorname{div} f)(t, x, \cdot)\|_{\mathbf{L}^{\infty}\left(\left[-U_{t}, U_{t}\right] ; \mathbb{R}\right)} \mathrm{d} x \mathrm{~d} t,
$$

where $U_{t}=\sup _{y \in \mathbb{R}^{N}}|u(t, y)|$.

The proof of this theorem is based on a good representation formula for the total variation and on the doubling variable method and is detailed in [18].

Remark 3.8. In some cases, we recover known estimates.

- When $f, F$ depend only on $u$, then $\kappa_{0}=\gamma$ and we recover the result of Remark $3.4: \operatorname{TV}(u(t)) \leq$ $\mathrm{TV}\left(u_{0}\right) e^{\gamma t}$

- When $f$ and $F$ do not depend on $u$, the equation reduces in fact to the ODE $\partial_{t} u=(F-\operatorname{div} f)(t, x)$, whose solution writes

Meanwhile, the bound above reduces to

$$
u(t, x)=u_{0}(x)+\int_{0}^{t}(F-\operatorname{div} f)(\tau, x) \mathrm{d} \tau .
$$

$$
\operatorname{TV}(u(t)) \leq \operatorname{TV}\left(u_{0}\right)+N W_{N} \int_{0}^{t} \int_{\mathbb{R}^{N}}|(F-\operatorname{div} f)(\tau, x)| \mathrm{d} x \mathrm{~d} \tau
$$

which is essentially what we expected, up to the coefficient $N W_{N}$.

Proof of Theorem 3.7. Let us admit the following proposition:

Proposition 3.9. Let $\mu \in \mathscr{C}_{c}^{\infty}\left(\mathbb{R}_{+} ; \mathbb{R}_{+}\right)$such that $\|\mu\|_{\mathbf{L}^{1}}=1$ and $\mu^{\prime}<0$ on $\mathbb{R}_{+}^{*}$. Let $u \in \mathbf{L}_{\text {loc }}^{1}\left(\mathbb{R}^{N}, \mathbb{R}\right)$. We define $\mu_{\lambda}(x)=\frac{1}{\lambda^{N}} \mu\left(\frac{\|x\|}{\lambda}\right)$. If there exists $C_{0}>0$ such that for all $\lambda>0$,

$$
\mathcal{I}(\lambda)=\frac{1}{\lambda} \int_{\mathbb{R}^{N}} \int_{\mathbb{R}^{N}}|u(x+y)-u(x)| \mu_{\lambda}(y) \mathrm{d} x \mathrm{~d} y \leq C_{0},
$$

then $u \in \mathbf{B V}\left(\mathbb{R}^{N}, \mathbb{R}\right)$ and

$$
C_{1} \operatorname{TV}(u)=\lim _{\lambda \rightarrow 0} \mathcal{I}(\lambda) \leq C_{0}
$$


with $C_{1}=\int_{\mathbb{R}^{N}}\left|y_{1}\right| \mu(\|y\|) \mathrm{d} y$.

This proposition leads us to introduce:

$$
\mathcal{F}(T, \lambda)=\int_{0}^{T} \int_{\mathbb{R}^{N}} \int_{B\left(x_{0}, R+M\left(T_{0}-t\right)\right)}|u(x+y)-u(x)| \mu_{\lambda}(y) \mathrm{d} x \mathrm{~d} y \mathrm{~d} t
$$

our goal being to estimate this quantity using the Kružkov doubling variable method.

Doubling variable method. Let us denote $u=u(t, x)$ and $v=u(s, y)$ for $(t, x),(s, y) \in \mathbb{R}_{+}^{*} \times \mathbb{R}^{N}$. For all $k, l \in \mathbb{R}$ and all test-function $\varphi=\varphi(t, x, s, y) \in \mathscr{C}_{c}^{1}\left(\left(\mathbb{R}_{+}^{*} \times \mathbb{R}^{N}\right)^{2} ; \mathbb{R}_{+}\right)$, we have, by definition of weak entropy solution (see Definition 3.1)

$$
\int_{\mathbb{R}_{+}^{*}} \int_{\mathbb{R}^{N}}\left[(u-k) \partial_{t} \varphi+(f(t, x, u)-f(t, x, k)) \nabla_{x} \varphi+(F(t, x, u)-\operatorname{div} f(t, x, k)) \varphi\right] \operatorname{sign}(u-k) \mathrm{d} x \mathrm{~d} t \geq 0
$$

and

$$
\int_{\mathbb{R}_{+}^{*}} \int_{\mathbb{R}^{N}}\left[(v-l) \partial_{s} \varphi+(f(s, y, v)-f(s, y, l)) \nabla_{y} \varphi+(F(s, y, v)-\operatorname{div} f(s, y, l)) \varphi\right] \operatorname{sign}(v-l) \mathrm{d} y \mathrm{~d} s \geq 0 .
$$

Let us choose $k=v(s, y)$ in (15) and integrate in $(s, y)$. Similarly, we choose $l=u(t, x)$ in $(16)$ and we integrate in $(t, x)$. Furthermore, we choose $\varphi(t, x, s, y)=\Psi(t-s, x-y) \Phi(t, x)$ and we sum to get

$$
\begin{gathered}
\int_{\mathbb{R}_{+}^{*}} \int_{\mathbb{R}^{N}} \int_{\mathbb{R}_{+}^{*}} \int_{\mathbb{R}^{N}} \operatorname{sign}(u-v)\left[(u-v) \Psi \partial_{t} \Phi+(f(t, x, u)-f(t, x, v)) \cdot(\nabla \Phi) \Psi\right. \\
+(f(s, y, v)-f(s, y, u)-f(t, x, v)+f(t, x, u)) \cdot(\nabla \Psi) \Phi \\
\quad+(F(t, x, u)-F(s, y, v)+\operatorname{div} f(s, y, u)-\operatorname{div} f(t, x, v)) \varphi] \mathrm{d} x \mathrm{~d} t \mathrm{~d} y \mathrm{~d} s \quad \geq 0 .
\end{gathered}
$$

We choose now $\mu$ and $\nu$ unit approximations and

$$
\Psi(t-s, x-y)=\frac{1}{\lambda^{N}} \mu\left(\frac{x-y}{\lambda}\right) \frac{1}{\eta} \nu\left(\frac{t-s}{\eta}\right)
$$

so that $\Psi$ converges to a Dirac in space and time when $\lambda, \eta \rightarrow 0$. We choose $\Phi$ such that $\Phi \rightarrow \mathbf{1}_{[0, T] \times B\left(x_{0}, R+M(T-t)\right)}$ when $\theta, \varepsilon \rightarrow 0$. The last step consists in estimating the integral (17) when $\eta, \theta, \varepsilon \rightarrow 0$, keeping the $\lambda$ fixed to make appear $\mathcal{F}$.

Estimate on $\mathcal{F}$. By the doubling variable method, we obtain

$$
\partial_{T} \mathcal{F}(T, \lambda) \leq \partial_{T} \mathcal{F}(0, \lambda)+C \lambda\left(\partial_{\lambda} \mathcal{F}(T, \lambda)+\frac{N}{\lambda} \mathcal{F}(T, \lambda)\right)+C^{\prime} \mathcal{F}(T, \lambda)+\lambda \int_{0}^{T} A(t) \mathrm{d} t,
$$

where

$$
\begin{aligned}
A(t) & =M_{1} \int_{\mathbb{R}^{N}}\|\nabla(F-\operatorname{div} f)(t, x, \cdot)\|_{\mathbf{L}^{\infty}(u)} \mathrm{d} x, & M_{1} & =\int_{\mathbb{R}^{N}}\|y\| \mu(\|y\|) \mathrm{d} y, \\
C^{\prime} & =N\left\|\nabla \partial_{u} f\right\|_{\mathbf{L}^{\infty}}+\left\|\partial_{u} F\right\|_{\mathbf{L}^{\infty}}, & C & =\left\|\nabla \partial_{u} f\right\|_{\mathbf{L}^{\infty}} .
\end{aligned}
$$

We use the hypothesis $u_{0} \in \mathbf{B V}\left(\mathbb{R}^{N}, \mathbb{R}\right)$ to get $\frac{1}{\lambda} \partial_{T} \mathcal{F}(0, \lambda) \leq M_{1} \mathrm{TV}\left(u_{0}\right)$, and we integrate with respect to time, obtaining:

$$
0 \leq \frac{M_{1}}{C} \mathrm{TV}\left(u_{0}\right)+\partial_{\lambda} \mathcal{F}(T, \lambda)+\frac{\alpha(T)}{\lambda} \mathcal{F}(T, \lambda)+\frac{1}{C} \int_{0}^{T} A(t) \mathrm{d} t
$$


where $\alpha(T)=N+\frac{C^{\prime}}{C}-\frac{1}{C T} \rightarrow_{T \rightarrow 0}-\infty$. Next, we choose $T$ so that $\alpha<-1$ and we integrate on $[\lambda,+\infty[$. We obtain:

$$
\mathcal{F}(T, \lambda) \leq \frac{\lambda}{-\alpha-1} \frac{M_{1}}{C} \operatorname{TV}\left(u_{0}\right)+\frac{\lambda}{C(-\alpha-1)} \int_{0}^{T} A(t) \mathrm{d} t .
$$

Besides, note that, thanks to the shape of $\mu$, we can find $K>0$ so that

$$
\partial_{\lambda} \mathcal{F}(T, \lambda)+\frac{N}{\lambda} \mathcal{F}=\int_{0}^{T} \int_{\mathbb{R}^{N}} \int_{B\left(x_{0}, R+M\left(T_{0}-t\right)\right)}|u(x+y)-u(x)| \mu_{\lambda}^{\prime}(y) \mathrm{d} x \mathrm{~d} y \mathrm{~d} t \leq \frac{K}{2 \lambda} \mathcal{F}(T, 2 \lambda) .
$$

Hence, we finally obtain

$$
\frac{1}{\lambda} \partial_{T} \mathcal{F}(T, \lambda) \leq \frac{1}{\lambda} \partial_{T} \mathcal{F}(0, \lambda)+\frac{C K+C^{\prime}}{(-\alpha-1) C}\left(M_{1} \mathrm{TV}\left(u_{0}\right)+\int_{0}^{T} A(t) \mathrm{d} t\right)+\int_{0}^{T} A(t) \mathrm{d} t .
$$

By Proposition 3.9 we have then $u(t) \in \mathbf{B V}\left(\mathbb{R}^{N}, \mathbb{R}\right)$. We can now divide (18) by $\lambda$ and make $\lambda \rightarrow 0$. Applying a standard Gronwall-type argument, we obtain the desired inequality on a small time interval. By iteration of the procedure, we extend the time interval to all $\mathbb{R}_{+}$.

\subsection{Scalar conservation law with a non-local flow}

Let us introduce the following definition:

Definition 3.10. We say that $\rho$ is a weak entropy solution to (1) with initial condition $\rho_{0} \in\left(\mathbf{L}^{\mathbf{1}} \cap \mathbf{L}^{\infty} \cap\right.$ $\mathbf{B V})\left(\mathbb{R}^{N}, \mathbb{R}\right.$ ) if it is a weak entropy solution (in the sense of Definition 3.1) of

$$
\partial_{t} \rho+\operatorname{Div}(\rho V(t, x))=0, \quad \rho(0, \cdot)=\rho_{0},
$$

where $V(t, x)=v(\eta * \rho(t, x)) \vec{\nu}(x)$.

We want to prove Theorem 2.1 and Theorem 2.3. Note that this proof adapts smoothly to prove Theorem 2.5 and 2.6. We use here the scheme proposed in Section 1 , choosing $X=\mathscr{C}^{0}\left([0, T], \mathbf{L}^{\mathbf{1}}\left(\mathbb{R}^{N}, \mathbb{R}\right)\right)$ at point (a). Point (b) is then satisfied by Kružkov theorem (see Theorem 3.2) and point (c) is satisfied by the stability estimate given by Theorem $3.6 \& 3.7$.

This scheme of proof can be also used for the other models: the proof of Theorem 2.5 for an orderly crowd is based on it, as well as Theorem 2.6 giving existence and uniqueness for the several populations models (6) and (8). For more details on these various theorems, refer to [5,7].

We use also this scheme of proof in order to prove existence and uniqueness of solution to equation (9), where we need furthermore stability results on the ODE (see [4]).

\subsubsection{Proof of Theorem 2.1}

Let us introduce the space

$$
X=\mathscr{C}^{0}\left([0, T], \mathbf{L}^{\mathbf{1}}\left(\mathbb{R}^{N} ; \mathbb{R}_{+}\right)\right) .
$$

Let $r \in X$ and $\rho \in X$ be the solution of (2) given by Kružkov Theorem (see Theorem 3.2). Note that, $u=0$ being a solution to (2), $\rho_{0} \geq 0$ implies $\rho(t) \geq 0$ for all $t \geq 0$ thanks to the maximal principle on scalar conservation laws [17, Theorem 3].

Remark 3.11. For the model (5) of orderly crowd, we can use the same kind of argument to have a uniform bound on the $\mathbf{L}^{\infty}$ norm. Indeed, if $u=1$ is a solution to the equation

$$
\partial_{t} \rho+\operatorname{Div}(\rho v(\rho) \vec{w}(x))=0
$$


then $\rho_{0} \leq 1$ implies $\rho(t) \leq 1$ for all $t \geq 0$. This fact allows us in this case to consider the space $X=$ $\mathscr{C}^{0}\left([0, T], \mathbf{L}^{\mathbf{1}}\left(\mathbb{R}^{N},[0,1]\right)\right)$. The same fact is also true for each density of the model (8) of orderly crowd with several populations. Indeed, the interaction between the equations is only in the non-local term; so, if we fix the nonlocal term, we have to deal with the same kind of scalar conservation law as (19). In particular, for each density $\rho_{i}$ the maximum principle gives us

$$
0 \leq \rho_{0, i} \leq 1 \quad \Rightarrow \quad 0 \leq \rho_{i}(t) \leq 1 \quad \text { for all } t \geq 0 .
$$

So finally, if there are $k$ populations, we have the following uniform bound on the total density density

$$
0 \leq \sum_{i=1}^{k} \rho_{i}(t) \leq k .
$$

Let us introduce the application $\mathscr{Q}: r \in X \rightarrow \rho \in X$. For $r_{1}, r_{2}$, we get by Theorem 3.6 and Theorem 3.7,

$$
\left\|\mathscr{Q}\left(r_{1}\right)-\mathscr{Q}\left(r_{2}\right)\right\|_{\mathbf{L}^{\infty}\left([0, T], \mathbf{L}^{1}\right)} \leq f(T)\left\|r_{1}-r_{2}\right\|_{\mathbf{L}^{\infty}\left([0, T], \mathbf{L}^{1}\right)},
$$

where $f$ is continuous, increasing, $f(0)=0$ and $f \rightarrow_{T \rightarrow \infty} \infty$. Consequently, we can choose $T$ small enough so that $f(T)=1 / 2$ and apply the Banach fixed point theorem for a small time. Iterating the process in time, we obtain global in time existence.

\subsubsection{Gâteaux derivative of the semi-group}

We prove now that the semi-group obtained in Theorem 2.1 (or 2.6) is Gâteaux-differentiable with respect to the initial condition. The following result is obtained by the use of Kružkov theory.

Note that the Gâteaux-differentiability is no longer possible for the more non-linear models of orderly crowd (5) and (8). Indeed an important tool below is that the equation (1) preserves the regularity of the initial condition, which is not satisfied by (5).

Definition 3.12. We say the application $S: \mathbf{L}^{\mathbf{1}}\left(\mathbb{R}^{N} ; \mathbb{R}\right) \rightarrow \mathbf{L}^{\mathbf{1}}\left(\mathbb{R}^{N} ; \mathbb{R}\right)$ is Gâteaux differentiable in $\mathbf{L}^{\mathbf{1}}$, in $\rho_{0} \in \mathbf{L}^{\mathbf{1}}$, in the direction $r_{0} \in \mathbf{L}^{\mathbf{1}}$ if there exists a linear continuous application $D S\left(\rho_{0}\right): \mathbf{L}^{\mathbf{1}} \rightarrow \mathbf{L}^{\mathbf{1}}$ such that

$$
\left\|\frac{S\left(\rho_{0}+h r_{0}\right)-S\left(\rho_{0}\right)}{h}-D S\left(\rho_{0}\right)\left(r_{0}\right)\right\|_{\mathbf{L}^{1}} \rightarrow_{h \rightarrow 0} 0 .
$$

We expect the Gâteaux derivative to be the solution of the linearized problem (4).

First, we prove that the linearized problem admits an entropy solution (see $[6,7]$ ).

Theorem 3.13. Assume that $v \in\left(\mathscr{C}^{4} \cap \mathbf{W}^{2, \infty}\right)(\mathbb{R}, \mathbb{R}), \vec{\nu} \in\left(\mathscr{C}^{3} \cap \mathbf{W}^{2,1} \cap \mathbf{W}^{2, \infty}\right)\left(\mathbb{R}^{N}, \mathbb{R}^{N}\right), \eta \in\left(\mathscr{C}^{3} \cap\right.$ $\left.\mathbf{W}^{\mathbf{2}, \infty}\right)\left(\mathbb{R}^{N}, \mathbb{R}_{+}\right)$. Let $\rho \in \mathscr{C}^{0}\left(\left[0, T_{\text {ex }}\left[; \mathbf{W}^{\mathbf{1}, \infty} \cap \mathbf{W}^{\mathbf{1}, \mathbf{1}}\left(\mathbb{R}^{N}, \mathbb{R}\right)\right), r_{0} \in\left(\mathbf{L}^{\mathbf{1}} \cap \mathbf{L}^{\infty}\right)\left(\mathbb{R}^{N} ; \mathbb{R}\right)\right.\right.$. Then the linearized problem (4) with initial condition $r_{0}$ admits a unique entropy solution $r \in \mathscr{C}^{0}\left(\left[0, T_{e x}\left[; \mathbf{L}^{\mathbf{1}}\left(\mathbb{R}^{N} ; \mathbb{R}\right)\right)\right.\right.$ and we denote $\Sigma_{t}^{\rho} r_{0}=r(t, \cdot)$.

If furthermore $r_{0} \in \mathbf{W}^{\mathbf{1}, \mathbf{1}}\left(\mathbb{R}^{N}, \mathbb{R}\right)$, then for all $t \in\left[0, T_{\text {ex }}\left[, r(t) \in \mathbf{W}^{\mathbf{1}, \mathbf{1}}\left(\mathbb{R}^{N}, \mathbb{R}\right)\right.\right.$.

The proof is similar to the one of Theorem 2.1 so we omit it.

Theorem 3.14. Assume that $v \in\left(\mathscr{C}^{4} \cap \mathbf{W}^{2, \infty}\right)(\mathbb{R}, \mathbb{R}), \vec{\nu} \in\left(\mathscr{C}^{3} \cap \mathbf{W}^{2,1} \cap \mathbf{W}^{2, \infty}\right)\left(\mathbb{R}^{N}, \mathbb{R}^{N}\right), \eta \in\left(\mathscr{C}^{3} \cap\right.$ $\left.\mathbf{W}^{\mathbf{2}, \infty}\right)\left(\mathbb{R}^{N}, \mathbb{R}_{+}\right)$. Let $\rho_{0} \in\left(\mathbf{W}^{\mathbf{1}, \infty} \cap \mathbf{W}^{\mathbf{2}, \mathbf{1}}\right)\left(\mathbb{R}^{N}, \mathbb{R}^{+}\right), r_{0} \in\left(\mathbf{W}^{\mathbf{1}, \mathbf{1}} \cap \mathbf{L}^{\infty}\right)\left(\mathbb{R}^{N}, \mathbb{R}\right)$.

Then, for every $t \geq 0$, the local semigroup of the pedestrian traffic problem is $\mathbf{L}^{\mathbf{1}}$ Gatteaux differentiable in the direction $r_{0}$ and

$$
D S_{t}\left(\rho_{0}\right)\left(r_{0}\right)=\Sigma_{t}^{S_{t} \rho_{0}} r_{0} .
$$


We prove this theorem following the same sketch of proof as above.

Proof. Let $\rho, \rho_{h}$ the solutions of the problem (1) with initial condition $\rho_{0}, \rho_{0}+h r_{0}$.

Let $r$ be the solution of the linearized problem (4), $r(0)=r_{0}$ and let $z_{h}=\rho+h r$ which then satisfies

$$
\partial_{t} z_{h}+\operatorname{Div}\left(z_{h}\left(v(\rho * \eta)+h v^{\prime}(\rho * \eta)(r * \eta)\right) \vec{\nu}(x)\right)=h^{2} \operatorname{Div}\left(r v^{\prime}(\rho * \eta)(r * \eta) \vec{\nu}(x)\right), \quad z_{h}(0)=\rho_{0}+h r_{0} .
$$

Then we use Theorem $3.6 \& 3.7$ to compare $\rho_{h}$ et $z_{h}$. We get:

$$
\begin{aligned}
\frac{1}{h}\left\|\rho_{h}-z_{h}\right\|_{\mathbf{L}^{\infty}\left(\left[0, T\left[, \mathbf{L}^{1}\right)\right.\right.} \leq & F(T)\left(\frac{1}{h}\left\|\rho_{h}-\rho\right\|_{\mathbf{L}^{\infty}\left(\mathbf{L}^{\mathbf{1}}\right)}^{2}+\frac{1}{h}\left\|\rho_{h}-z_{h}\right\|_{\mathbf{L}^{\infty}\left(\mathbf{L}^{\mathbf{1}}\right)}\right) \\
& +h C(\beta) T e^{C(\beta) T}\|r\|_{\mathbf{L}^{\infty}\left(\mathbf{W}^{1,1}\right)}\|r\|_{\mathbf{L}^{\infty}\left(\mathbf{L}^{\mathbf{1}}\right)},
\end{aligned}
$$

with $F$ increasing and $F(0)=0$. Then, we choose $T$ small enough so that $F(T)=1 / 2$. Besides, applying Theorem 3.6 to compare $\rho$ and $\rho_{h}$ and using Gronwall lemma, we obtain that $\frac{1}{h}\left\|\rho_{h}-\rho\right\|_{\mathbf{L}^{\infty}\left(\mathbf{L}^{1}\right)}$ remains bounded when $h \rightarrow 0$.

Finally, we can take the limit $h \rightarrow 0$ and obtain the desired result. Once again, we have here a local in time result that can be extended to $\mathbb{R}_{+}$by a standard iteration procedure.

\section{Using the Optimal tRAnsport theORY}

In this section, we want to prove Theorem 2.2, giving existence and uniqueness of weak measure solution to (1) and (6). In a more general settings, we consider the system:

$$
\begin{aligned}
\partial_{t} \rho_{i}+\operatorname{Div}\left(\rho_{i} V_{i}\left(x, \rho_{1} * \eta_{i, 1}, \ldots, \rho_{k} * \eta_{i, k}\right)\right) & =0, & (t, x) & \in \mathbb{R}_{+} \times \mathbb{R}^{N} \\
\rho_{i}(0) & =\bar{\rho}_{i}, & i & \in\{1, \ldots, k\} .
\end{aligned}
$$

in which the coupling between the equations is only present through the nonlocal term. Fixing the nonlocal term, we obtain a system of decoupled continuity equations:

$$
\left\{\begin{array}{l}
\partial_{t} \rho_{1}+\operatorname{Div}\left(\rho_{1} b_{1}(t, x)\right)=0, \\
\cdots \\
\partial_{t} \rho_{k}+\operatorname{Div}\left(\rho_{k} b_{k}(t, x)\right)=0,
\end{array}\right.
$$

where $b_{1}, \ldots, b_{k}$ are regular with respect to $x$.

\subsection{Existence and uniqueness of weak measure solutions}

Let us remind the following definitions of weak measure solution, but also of Lagrangian solution.

Definition 4.1. We say that $\rho \in \mathbf{L}^{\infty}\left([0, T], \mathcal{M}^{+}\left(\mathbb{R}^{N}\right)^{k}\right)$ is a measure solution of (20) with initial condition $\bar{\rho} \in \mathcal{M}^{+}\left(\mathbb{R}^{N}\right)^{k}$ if, for all test-function $\varphi \in \mathscr{C}_{c}^{\infty}(]-\infty, T\left[\times \mathbb{R}^{N}, \mathbb{R}\right)$

$$
\int_{0}^{T} \int_{\mathbb{R}^{N}}\left[\partial_{t} \varphi+V_{i}\left(x, \rho * \eta_{i}\right) \cdot \nabla \varphi\right] \mathrm{d} \rho_{t}^{i}(x) \mathrm{d} t+\int_{\mathbb{R}^{N}} \varphi(0, x) \mathrm{d} \bar{\rho}_{i}(x)=0 .
$$

Definition 4.2. We say that $\rho \in \mathbf{L}^{\infty}\left([0, T], \mathcal{M}^{+}\left(\mathbb{R}^{N}\right)^{k}\right)$ is a Lagrangian solution with initial condition $\bar{\rho} \in$ $\mathcal{M}^{+}\left(\mathbb{R}^{N}\right)^{k}$ if there exists an ODE flow $X^{i}:[0, T] \times \mathbb{R}^{N} \rightarrow \mathbb{R}^{N}$, solution of

$$
\left\{\begin{aligned}
\frac{\mathrm{d} X^{i}}{\mathrm{~d} t}(t, x) & =V_{i}\left(X^{i}(t, x), \rho_{t} * \eta^{i}\left(X^{i}(t, x)\right)\right) \\
X^{i}(0, x) & =x
\end{aligned}\right.
$$


and such that $\rho_{t}^{i}=X_{t \sharp}^{i} \bar{\rho}^{i}$ where $X_{t}^{i}: \mathbb{R}^{N} \rightarrow \mathbb{R}^{N}$ is the map defined as $X_{t}^{i}(x)=X^{i}(t, x)$ for any $(t, x) \in \mathbb{R}_{+} \times \mathbb{R}^{N}$.

Definition 4.3. Let $\mu$ be measure on $\Omega$ and $T: \Omega \rightarrow \Omega^{\prime}$ a measurable map. Then $T_{\sharp} \mu$ is the push-forward of $\mu$ if for any $\varphi \in \mathscr{C}_{c}^{0}\left(\Omega^{\prime}\right)$,

$$
\int_{\Omega^{\prime}} \varphi(x) \mathrm{d} T_{\sharp} \mu(x)=\int_{\Omega} \varphi(T(y)) \mathrm{d} \mu(y) .
$$

If $T_{\sharp} \mathrm{d} \mu(x)=f(x) \mathrm{d} x$ and $\mathrm{d} \mu(y)=g(y) \mathrm{d} y$, and $T$ is a $\mathscr{C}^{1}$-diffeomorphism, then we have the change of variable formula

$$
g(x)=f(T(x))|\operatorname{det}(\nabla T(x))| .
$$

Instead of proving directly Theorem 2.2 (or Theorem 2.7), we prove first the existence and uniqueness of Lagrangian solutions.

Theorem 4.4. Let $\bar{\rho} \in \mathcal{M}^{+}\left(\mathbb{R}^{N}, \mathbb{R}^{k}\right)$. Let us assume that $V \in\left(\mathbf{L}^{\infty} \cap \operatorname{Lip}\right)\left(\mathbb{R}^{N} \times \mathbb{R}^{k}, \mathbb{R}^{N \times k}\right)$ and that $\eta \in$ $\left(\mathbf{L}^{\infty} \cap \operatorname{Lip}\right)\left(\mathbb{R}^{N}, \mathbb{R}^{k \times k}\right)$. Then there exists a unique Lagrangian solution to system (20) with initial condition $\bar{\rho}$.

Remark 4.5. Note that a Lagrangian solution is also a weak measure solution. Indeed, if $\rho_{t}^{i}=X_{t \sharp}^{i} \bar{\rho}^{i}$ is a Lagrangian solution, by the definition 4.3 of push-forward, we get, with $\varphi \in \mathscr{C}_{c}^{\infty}(]-\infty, T\left[\times \mathbb{R}^{N}, \mathbb{R}\right)$

$$
\begin{aligned}
& \int_{0}^{T} \int_{\mathbb{R}^{N}}\left[\partial_{t} \varphi+V_{i}\left(x, \rho * \eta_{i}\right) \cdot \nabla \varphi\right] \mathrm{d} \rho_{t}^{i}(x) \mathrm{d} t \\
= & \int_{0}^{T} \int_{\mathbb{R}^{N}}\left[\partial_{t} \varphi\left(t, X_{t}^{i}(x)\right)+V_{i}\left(X_{t}^{i}(x), \rho * \eta_{i}\left(X_{t}^{i}(x)\right)\right) \cdot \nabla \varphi\left(t, X_{t}^{i}(x)\right)\right] \mathrm{d} \bar{\rho}^{i}(x) \mathrm{d} t \\
= & \int_{0}^{T} \int_{\mathbb{R}^{N}}\left[\partial_{t} \varphi\left(t, X_{t}^{i}(x)\right)+\frac{\mathrm{d} X^{i}(t, x)}{\mathrm{d} t} \cdot \nabla \varphi\left(t, X_{t}^{i}(x)\right)\right] \mathrm{d} \bar{\rho}^{i}(x) \mathrm{d} t \\
= & \int_{0}^{T} \int_{\mathbb{R}^{N}} \frac{\mathrm{d}}{\mathrm{d} t}\left(\varphi\left(t, X^{i}(t, x)\right)\right) \mathrm{d} \rho_{t}^{i}(x) \mathrm{d} t \\
= & -\int_{\mathbb{R}^{N}} \varphi(0, x) \mathrm{d} \bar{\rho}^{i}(x) .
\end{aligned}
$$

Remark 4.6. We recover the results of Theorem 2.1, except the continuity in time. Indeed, Lagrangian solutions preserve the regularity of the initial condition along the time, and in particular if $\bar{\rho} \in \mathbf{L}^{\mathbf{1}}\left(\mathbb{R}^{N},\left(\mathbb{R}^{+}\right)^{k}\right)$ then $\rho \in \mathbf{L}^{\infty}\left(\mathbb{R}^{+}, \mathbf{L}^{\mathbf{1}}\left(\mathbb{R}^{N},\left(\mathbb{R}^{+}\right)^{k}\right)\right)$ and we have

$$
\|\rho(t)\|_{\mathbf{L}^{1}}=\|\bar{\rho}\|_{\mathbf{L}^{1}} .
$$

If moreover $\bar{\rho} \in\left(\mathbf{L}^{\mathbf{1}} \cap \mathbf{L}^{\infty}\right)\left(\mathbb{R}^{N},\left(\mathbb{R}^{+}\right)^{k}\right)$, then $\rho(t) \in \mathbf{L}^{\infty}$ for all $t \geq 0$ and we have the estimate

$$
\|\rho(t)\|_{\mathbf{L}^{\infty}} \leq\|\bar{\rho}\|_{\mathbf{L}^{\infty}} e^{C t},
$$

where $C$ depends on $\|\bar{\rho}\|_{\mathcal{M}}, V$ and $\eta$.

We conclude the proof of Theorem 2.2 stating the uniqueness of measure solutions, so that the Lagrangian solution obtained is also the unique measure solution.

Proposition 4.7. Under the same set of hypotheses as Theorem 4.4, there exists at most one weak measure solution to (20).

\subsection{Proof of Theorem 4.4}

Once again, the proof is based on the scheme described in Section 1. We consider below probability measures instead. Note that we are considering measure of total mass 1, but, by rescaling, we can also consider positive measures of fixed total mass $\left\|\bar{\rho}^{i}\right\|_{\mathcal{M}}=M_{i}$, for every $i$. 
(a) Let us first introduce a space $X=\mathbf{L}^{\infty}\left([0, T], \mathcal{P}\left(\mathbb{R}^{N}\right)^{k}\right)$. We equip this space with the distance $d(\mu, \nu)=$ $\sup _{t \in[0, T]} \mathcal{W}_{1}\left(\mu_{t}, \nu_{t}\right)$, the Wasserstein distance $\mathcal{W}_{1}$ being defined as follow:

Definition 4.8. Let $\mu, \nu \in \mathcal{P}\left(\mathbb{R}^{N}\right)$. Let us denote $\mathbb{P}_{x}: \mathbb{R}^{d} \times \mathbb{R}^{d} \rightarrow \mathbb{R}^{d}$ the projection on the first coordinate; that is, for any $(u, v) \in \mathbb{R}^{d} \times \mathbb{R}^{d}, \mathbb{P}_{x}(u, v)=u$. In a similar way, $\mathbb{P}_{y}: \mathbb{R}^{d} \times \mathbb{R}^{d} \rightarrow \mathbb{R}^{d}$ is the projection on the second coordinate; that is, for any $(u, v) \in \mathbb{R}^{d} \times \mathbb{R}^{d}, \mathbb{P}_{y}(u, v)=v$. We denote $\Xi(\mu, \nu)$ the set of plans, that is

$$
\Xi(\mu, \nu)=\left\{\gamma \in \mathcal{P}\left(\mathbb{R}^{N} \times \mathbb{R}^{N}\right): \mathbb{P}_{x \sharp} \gamma=\mu \text { and } \mathbb{P}_{y \sharp} \gamma=\nu\right\} .
$$

The Wasserstein distance of order one between $\mu$ and $\nu$ is

$$
W_{1}(\mu, \nu)=\inf _{\gamma \in \Xi(\mu, \nu)} \int_{\mathbb{R}^{N} \times \mathbb{R}^{N}}|x-y| \mathrm{d} \gamma(x, y) .
$$

Let $\rho=\left(\rho^{1}, \ldots, \rho^{k}\right), \sigma=\left(\sigma^{1}, \ldots, \sigma^{k}\right) \in \mathcal{P}\left(\mathbb{R}^{N}\right)^{k}$. The Wasserstein distance of order one between $\rho$ and $\sigma$, denoted $\mathcal{W}_{1}(\rho, \sigma)$, as $\mathcal{W}_{1}(\rho, \sigma)=\sum_{i=1}^{k} W_{1}\left(\rho^{i}, \sigma^{i}\right)$.

Let us recall the following duality formula:

Proposition 4.9 (cf. Villani [23, p. 207]). Let f,g be two probability measures. The Wasserstein distance of order one between $f$ and $g$ satisfies

$$
W_{1}(f, g)=\sup _{\operatorname{Lip}(\varphi) \leq 1} \int_{\mathbb{R}^{d}} \varphi(x)(\mathrm{d} f(x)-\mathrm{d} g(x)) .
$$

(b) Existence of Lagrangian solutions.

Let $r \in \mathbf{L}^{\infty}\left([0, T], \mathcal{P}\left(\mathbb{R}^{N}\right)^{k}\right)$. Define $b_{i}(t, x)=V_{i}\left(x, r_{t} * \eta_{i}\right) \in \mathbf{L}^{\infty}\left([0, T], \mathbf{W}^{\mathbf{1}, \infty}\left(\mathbb{R}^{N}\right)^{k}\right)$. Let us consider the equation

$$
\partial_{t} \rho_{i}+\operatorname{Div}\left(\rho_{i} b_{i}(t, x)\right)=0 .
$$

Let $\rho_{i}=X_{t \sharp \bar{\rho}}$ be the Lagrangian solution of (21). Then the application

$$
\mathscr{T}: r \in \mathbf{L}^{\infty}\left([0, T], \mathcal{P}\left(\mathbb{R}^{N}\right)^{k}\right) \mapsto \rho \in \mathbf{L}^{\infty}\left([0, T], \mathcal{P}\left(\mathbb{R}^{N}\right)^{k}\right) .
$$

is well-defined.

(c) Stability estimate.

Proposition 4.10. Let $\bar{\rho}, \bar{\sigma} \in \mathcal{P}\left(\mathbb{R}^{N}\right)$ and $r, s \in \mathscr{C}^{0}\left([0, T], \mathcal{P}\left(\mathbb{R}^{N}\right)\right)$. Let $V \in\left(\mathbf{L}^{\infty} \cap \operatorname{Lip}\right)\left(\mathbb{R}^{N} \times \mathbb{R}^{k}, \mathbb{R}^{N}\right)$, $\eta, \nu \in\left(\mathbf{L}^{\infty} \cap \operatorname{Lip}\right)\left(\mathbb{R}^{N}, \mathbb{R}\right)$. If $\rho$ and $\sigma$ are Lagrangian solutions of

$$
\begin{array}{ll}
\partial_{t} \rho+\operatorname{Div}(\rho V(x, r * \eta))=0, & \rho(0, \cdot)=\bar{\rho} \\
\partial_{t} \sigma+\operatorname{Div}(\sigma V(x, s * \eta))=0, & \sigma(0, \cdot)=\bar{\sigma} .
\end{array}
$$

We have the estimate:

$$
\mathcal{W}_{1}\left(\rho_{T}, \sigma_{T}\right) \leq e^{C T} \mathcal{W}_{1}(\bar{\rho}, \bar{\sigma})+T e^{C T} C^{\prime} \sup _{t \in[0, T]} \mathcal{W}_{1}\left(r_{t}, s_{t}\right)
$$

where $C=\operatorname{Lip}_{x}(V)+\operatorname{Lip}_{r}(V) \operatorname{Lip}(\eta)\|\bar{\rho}\|_{\mathcal{M}}+\operatorname{Lip}_{r}(V) \operatorname{Lip}(\eta)\|\bar{\rho}\|_{\mathcal{M}}$ and $C^{\prime}=\operatorname{Lip}_{r}(V) \operatorname{Lip}(\eta)\|\bar{\rho}\|_{\mathcal{M}}$.

Proof. Let $X, Y$ be the ODE flows associated with $\rho, \sigma$. Let $\gamma_{0} \in \Xi(\bar{\rho}, \bar{\sigma})$. Define

$$
X_{t} \bowtie Y_{t}:(x, y) \mapsto(X(x), Y(y)) .
$$


Then $\gamma_{t}=\left(X_{t} \bowtie Y_{t}\right)_{\sharp} \gamma_{0} \in \Xi\left(\rho_{t}, \sigma_{t}\right)$. Let us introduce

$$
Q(t)=\int_{\mathbb{R}^{N} \times \mathbb{R}^{N}}|x-y| \mathrm{d} \gamma_{t}(x, y)=\int_{\mathbb{R}^{N} \times \mathbb{R}^{N}}\left|X_{t}(x)-Y_{t}(y)\right| \mathrm{d} \gamma_{0}(x, y) .
$$

Then $Q$ is a Lipschitz function and

$$
Q^{\prime}(t) \leq \int_{\mathbb{R}^{N} \times \mathbb{R}^{N}}\left|V\left(X_{t}(x), r_{t} * \eta\left(X_{t}(x)\right)\right)-V\left(Y_{t}(y), s_{t} * \eta\left(Y_{t}(x)\right)\right)\right| \mathrm{d} \gamma_{0}(x, y) .
$$

By triangular inequality, we obtain

$$
Q^{\prime}(t) \leq\left(\operatorname{Lip}_{x}(V)+\operatorname{Lip}_{r}(V) \operatorname{Lip}\left(r_{t} * \eta\right)\right) Q(t)+\operatorname{Lip}_{r}(V) \int_{\mathbb{R}^{N} \times \mathbb{R}^{N}}\left|\left(r_{t}-s_{t}\right) * \eta\left(Y_{t}(y)\right)\right| \mathrm{d} \gamma_{0}(x, y) .
$$

Note besides that, thanks to Proposition 4.9, we have

$$
\left(r_{t}^{i}-s_{t}^{i}\right) * \eta(z)=\int_{\mathbb{R}^{N}} \eta(z-\zeta)\left(\mathrm{d} r_{t}^{i}(\zeta)-d s_{t}^{i}(\zeta)\right) \leq \operatorname{Lip}(\eta) W_{1}\left(r_{t}^{i}, s_{t}^{i}\right) .
$$

Integrating, we get

$$
Q(t) \leq Q(0) e^{C t}+t C^{\prime} e^{C t} \sup _{\tau} \mathcal{W}_{1}\left(r_{\tau}, s_{\tau}\right) .
$$

where $C=\operatorname{Lip}_{x}(V)+\operatorname{Lip}_{r}(V)\left\|r_{t}\right\|_{\mathcal{M}^{\mathcal{M}}} \operatorname{Lip}(\eta), C^{\prime}=\operatorname{Lip}_{r}(V) \operatorname{Lip}(\eta)\|\bar{\rho}\|_{\mathcal{M}}$.

We conclude taking $\gamma_{0}$ in an optimal way so that $Q(0)=\mathcal{W}_{1}\left(\rho_{0}, \sigma_{0}\right)$ and using the inequality

$$
\mathcal{W}_{1}\left(\rho_{t}, \sigma_{t}\right) \leq Q(t)
$$

The stability estimate above allows us to apply Banach fixed point Theorem for $T$ small enough and to obtain Lagrangian solutions to (20).

\subsection{Measure solutions are Lagrangian solutions}

Proof of Proposition 4.7. Let $\rho$ be a measure solution of (20). Let $b=V(x, \rho * \eta)$ and denote $\sigma$ the Lagrangian solution associated to $\partial_{t} \sigma+\operatorname{Div}(\sigma b)=0$ with $\sigma(0)=\bar{\rho}$.

Then, by Remark 4.5, $\delta=\rho-\sigma$ is a measure solution of $\partial_{t} \delta+\operatorname{Div}(\delta b)=0$, with $\delta(0)=0$. That is to say, for any $\varphi \in \mathscr{C}_{c}^{\infty}(]-\infty, T\left[\times \mathbb{R}^{N}, \mathbb{R}\right)$,

$$
\int_{0}^{T} \int_{\mathbb{R}^{N}}\left(\partial_{t} \varphi+b^{i}(t, x) \cdot \nabla \varphi\right) \mathrm{d} \delta_{t} \mathrm{~d} t=0 .
$$

Let $\psi \in \mathscr{C}_{c}^{0}(]-\infty, T\left[\times \mathbb{R}^{d}, \mathbb{R}\right)$. Since $b^{i} \in \mathbf{L}^{\infty}\left([0, T] \times \mathbb{R}^{d}, \mathbb{R}^{d}\right)$ is Lipschitz in $x$, by computation along the characteristics, we can find $\varphi \in \mathscr{C}_{c}^{1}(]-\infty, T\left[\times \mathbb{R}^{d}, \mathbb{R}\right)$ so that $\psi=\partial_{t} \varphi+b^{i}(t, x) \cdot \nabla \varphi$. Hence, for any $\psi \in$ $\mathscr{C}_{c}^{0}(]-\infty, T\left[\times \mathbb{R}^{d}, \mathbb{R}\right)$, we have $\int_{0}^{T} \int_{\mathbb{R}^{d}} \psi \mathrm{d} \delta_{t} \mathrm{~d} t=0$, which implies $\delta \equiv 0$ a.e., and so $\rho \equiv \sigma$ a.e. Consequently, we have also $b^{i}(t, x)=V^{i}\left(t, x, \sigma * \eta^{i}\right)$, and $\sigma=\rho$ is finally a Lagrangian solution of (20). 


\section{CONCLUSiOn}

In the Kružkov framework, we are able to prove existence and uniqueness of weak entropy solution for the equation $\partial_{t} \rho+\operatorname{Div}(\rho V(x, \rho, \rho * \eta))=0$. Furthermore, we can prove uniform bound in $\mathbf{L}^{\infty}$ if $V=v(\rho) \vec{W}(x, \rho * \eta)$, with $v(1)=0$. However, the required hypotheses are very strong: we need indeed $V \in \mathscr{C}^{2} \cap \mathbf{W}^{\mathbf{2}, \mathbf{1}} \cap \mathbf{W}^{\mathbf{2}, \infty}$.

In the optimal transport theory framework, we can treat only equations such that $\partial_{t} \rho+\operatorname{Div}(\rho V(x, \rho *$ $\eta))=0$. For this equation, we have only $\mathbf{L}^{\infty}$ bound that are exponentially growing in time. The hypotheses are nevertheless weaker since we only ask $V \in \mathbf{L i p} \cap \mathbf{L}^{\infty}$, but we are no longer able to prove the Gâteauxdifferentiability of the semi-group.

\section{REFERENCES}

[1] C. Appert-Rolland, P. Degond, and S. Motsch. Two-way multi-lane traffic model for pedestrians in corridors. Networks and Heterogeneous Media, 6(3):351-381, 2011.

[2] N. Bellomo and C. Dogbe. On the modeling of traffic and crowds: a survey of models, speculations, and perspectives. SIAM Rev., 53(3):409-463, 2011.

[3] F. Bouchut and B. Perthame. Kružkov's estimates for scalar conservation laws revisited. Trans. Amer. Math. Soc., 350(7):28472870,1998

[4] R. Colombo and M. Lécureux-Mercier. An analytical framework to describe the interactions between individuals and a continuum. Journal of Nonlinear Science, 22:39-61, 2012.

[5] R. M. Colombo, M. Garavello, and M. Lécureux-Mercier. A class of non-local models for pedestrian traffic. Mathematical Models and Methods in Applied Sciences (M3AS), 22, 2010.

[6] R. M. Colombo, M. Herty, and M. Mercier. Control of the continuity equation with a non local flow. ESAIM Control Optim. Calc. Var., 17(2):353-379, 2011.

[7] R. M. Colombo and M. Lécureux-Mercier. Nonlocal crowd dynamics models for several populations. Acta Mathematica Scientia, 32(1):177 - 196, 2012.

[8] V. Coscia and C. Canavesio. First-order macroscopic modelling of human crowd dynamics. Math. Models Methods Appl. Sci., 18(suppl.):1217-1247, 2008.

[9] G. Crippa and M. Lécureux-Mercier. Existence and uniqueness of measure solutions for a system of continuity equations with non-local flow. NoDEA : Nonlinear Differential Equations and Applications, pages 1-15, 2012.

[10] W. Daamen and S. Hoogendoorn. Experimental research of pedestrian walking behavior. In Transportation Research Board annual meeting 2003, pages 1-16. National Academy Press, 2007.

[11] M. Di Francesco, P. A. Markowich, J.-F. Pietschmann, and M.-T. Wolfram. On the Hughes' model for pedestrian flow: the one-dimensional case. J. Differential Equations, 250(3):1334-1362, 2011.

[12] D. Helbing and A. Johansson. Pedestrian, crowd and evacuation dynamics. Encyclopedia of Complexity and Systems Science, pages 6476-6495, 2010.

[13] D. Helbing, A. Johansson, and H. Z. Al-Abideen. Dynamics of crowd disasters: An empirical study. Phys. Rev. E, 75:046109, Apr 2007.

[14] D. Helbing, P. Molnár, I. Farkas, and K. Bolay. Self-organizing pedestrian movement. Environment and Planning B: Planning and Design, 28:361-383, 2001.

[15] R. L. Hughes. A continuum theory for the flow of pedestrians. Transportation Research Part B: Methodological, 36(6):507-535, 2002.

[16] R. L. Hughes. The flow of human crowds. Annual Review of Fluid Mechanics, 35:169-182, 2003.

[17] S. N. Kružkov. First order quasilinear equations with several independent variables. Mat. Sb. (N.S.), 81 (123):228-255, 1970.

[18] M. Lécureux-Mercier. Improved stability estimates for general scalar conservation laws. J. Hyperbolic Differ. Equ., 8(4):727$757,2011$.

[19] B. J. Lucier. A moving mesh numerical method for hyperbolic conservation laws. Math. Comp., 1986.

[20] B. Maury, A. Roudneff-Chupin, and F. Santambrogio. A macroscopic crowd motion model of the gradient-flow type. Mathematical Models and Methods in Applied Sciences, 20(10):1787-1821, 2010.

[21] B. Maury, A. Roudneff-Chupin, F. Santambrogio, and J. Venel. Handling congestion in crowd motion modeling. 2011.

[22] B. Piccoli and A. Tosin. Time-evolving measures and macroscopic modeling of pedestrian flow. Archive for Rational Mechanics and Analysis, pages 1-32, 2010.

[23] C. Villani. Topics in optimal transportation, volume 58 of Graduate Studies in Mathematics. American Mathematical Society, Providence, RI, 2003. 